\title{
Mixed network calculus
}

\section{Yegor Zenkevich}

SISSA, via Bonomea 265, 34136 Trieste, Italy

INFN, Sezione di Trieste, IGAP, via Beirut 2/1, 34151 Trieste, Italy

ITEP, Bolshaya Cheremushkinskaya street 25, 117218 Moscow, Russia

ITMP MSU, Leninskie gory 1, 119991 Moscow, Russia

MIPT, Institutskii pereulok 9, 141700, Dolgoprudny, Russia

E-mail: yegor.zenkevich@gmail.com

ABSTRACT: We show how to combine higgsed topological vertices introduced in [7] with conventional refined topological vertices. We demonstrate that the extended formalism describes very general interacting D5-NS5-D3 brane systems. In particular, we introduce new types of intertwining operators of Ding-Iohara-Miki algebra between different types of Fock representations corresponding to the crossings of NS5 and D5 branes. As a byproduct we obtain an algebraic description of the Hanany-Witten brane creation effect, give an efficient recipe to compute the brane factors in $3 d \mathcal{N}=2$ and $\mathcal{N}=4$ quiver gauge theories and demonstrate how $3 d S$-duality appears in our setup.

Keywords: Conformal and W Symmetry, D-branes, Topological Strings, Supersymmetric Gauge Theory

ARXIV EPRINT: 2012.15563 


\section{Contents}

1 Introduction 1

$\begin{array}{lll}2 & \text { D3 brane joins D5 brane } & 6\end{array}$

$3(p, q)$-string joins D3 brane: the brane nature of DIM representations 7

4 5-brane crossings $\quad 9$

4.1 5-brane crossing arise from degenerate resolved picture $\quad 9$

$\begin{array}{ll}4.2 & 5 \text {-brane crossing is the DIM Lax operator } \\ \end{array}$

$\begin{array}{lll}\text { 4.2.1 Lax operator from two 5-brane junctions } & 10\end{array}$

4.2.2 Lax operator from the universal DIM $R$-matrix 11

$\begin{array}{lll}4.3 & \text { Functoriality of the crossing } & 14\end{array}$

$\begin{array}{ll}\text { 4.4 Crossing between two 5-branes of different types } & 16\end{array}$

$\begin{array}{ll}\text { 4.5 Hanany-Witten D3 brane creation } & 17\end{array}$

$\begin{array}{ll}\text { 4.6 Crossing of a NS5 brane and a D3 brane } & 18\end{array}$

$\begin{array}{lll}\text { 4.6.1 } & \mathrm{NS} 5_{q, t^{-1}} \text { and } \mathrm{D} 3_{q} \text { branes } & 18\end{array}$

$\begin{array}{ll}\text { 4.6.2 } \mathrm{NS} 5_{q, t^{-1}} \text { and } \mathrm{D} 3_{t / q} \text { branes } & 19\end{array}$

$\begin{array}{lll}4.7 & \text { Hanany-Witten }(p, q) \text {-string creation } & 19\end{array}$

$\begin{array}{lll}4.8 \text { Crossing of Fock and MacMahon representations } & 21\end{array}$

5 Higgsed vertices from refined topological vertices $\quad 21$

$\begin{array}{lll}6 & T[\mathrm{SU}(N)] \text { vs } F T[\mathrm{SU}(N)] \text { partition functions and brane factors } & \mathbf{2 3}\end{array}$

6.1 Crossings commute with screening charges 24

6.2 Brane factors from commutation relations between crossings and intertwiners 24

$\begin{array}{lll}6.3 & S \text {-duality and spectral duality } & 26\end{array}$

6.4 Dualities in the double elliptic system 26

$\begin{array}{lll}7 & \text { Conclusions and discussions } & 27\end{array}$

$\begin{array}{ll}\text { A Intertwining property of the D3-D5 junction } & 28\end{array}$

\section{Introduction}

Branes and algebras are related. Very generally, if a brane has some other branes bound to it, then usually these states form a representation of a "brane algebra". Probably the most well-known of such setups is the Nakajima construction of the action of an affine Lie algebra on the moduli space of instantons on $\mathbb{R}^{4}[1-3]$. The development of the same ideas lead to the AGT relation [4-6]. In this paper we will consider one example of a brane algebra, 
namely the Ding-Iohara-Miki (DIM) algebra $[8,9]$ and show that it describes surprisingly many brane pictures. We will mostly focus on $3 d$ theories living on D3 branes, but many different objects will also appear naturally in our treatment.

The original motivation for this investigation [7] was to reinterpret the brane constructions and dualities of $3 d$ gauge theories with $\mathcal{N}=2$ and $\mathcal{N}=4$ supersymmetry using the "algebraic engineering" approach. By this term we mean a dictionary between brane pictures ${ }^{1}$ and networks of intertwiners of certain typically infinite-dimensional algebras. In particular, DIM algebra is a quantum toroidal algebra $\left.U_{q, t}\left(\widehat{\hat{\mathfrak{g l}}}_{1}\right)\right),{ }^{2}$ so it is "doubly infinite": the generators span an integer plane $\mathbb{Z}^{2}$. Related constructions for other algebras have been considered in [14-16], and a different perspective on them was proposed in [17-23]. In what follows we will frequently use the notations and conventions for DIM algebra and its representations taken from appendix A of [7] without referring to particular sections. So by default one should look up in there all the objects related DIM algebra used below.

The algebraic engineering approach that we will pursue here originated from the interpretation [12] of the refined topological vertex [10,11] as an intertwining operator of the DIM algebra. In this approach the lines on the brane pictures correspond to representations of the algebra while the intertwining operators between representations play the role of brane junctions. One draws Fock representations of the DIM algebra as (solid) lines of rational slopes lying in a $2 d$ plane:

$$
u f=\mathcal{F}_{q, t^{-1}}^{(p, q)}(u)
$$

where $u$ denotes the spectral parameter. In the brane language these lines can be understood as $(p, q) 5$-branes ${ }^{3}$ of Type IIB string theory forming a brane web. Their slope in the picture is determined by their $(p, q)$-charges as follows from supersymmetry constraints. The $\mathrm{SL}(2, \mathbb{Z})$ automorphism group of the DIM algebra acts on the plane of the picture and correspondingly on the 5 -brane $(p, q)$-charges. It is therefore identified with the $S$-duality group of the Type IIB string theory.

The triple junction of 5 -branes corresponds to the intertwining operators $\Psi_{q, t^{-1}}$ : $\mathcal{F}_{q, t^{-1}}^{(0,1)}(w) \otimes \mathcal{F}_{q, t^{-1}}^{(1,0)}(u) \rightarrow \mathcal{F}_{q, t^{-1}}^{(1,1)}(-u w):$

$$
\Psi_{q, t^{-1}}=w_{-u w}^{w \downarrow} u
$$

There is also the dual intertwiner $\Psi^{*}$ acting from a single Fock representation to a pair of Fock representations. The explicit expressions for the intertwiners $\Psi$ and $\Psi^{*}$ are given in $[12]$.

\footnotetext{
${ }^{1}$ Through a chain of dualities the same pictures can sometimes be interpreted as purely geometric backgrounds, e.g. toric diagrams of toric Calabi-Yau three-folds.

${ }^{2}$ A generalization of the construction to $U_{q, t}\left(\widehat{\widehat{\mathfrak{g l}}}_{N}\right)$ is also available [13].

${ }^{3}$ There is an unpleasant clash in our notation: $q$ denotes one of the charges of the 5 -brane and also the $\Omega$-background deformation parameter. However, both conventions are so entrenched in the literature that we do not dare to change them and can only hope that there are not too many opportunities for confusion.
} 
The charges of the 5 -branes have to be conserved at the junction so a $\left(r_{1}, s_{1}\right)$ and $\left(r_{2}, s_{2}\right)$ branes join into a $\left(r_{1}+r_{2}, s_{1}+s_{2}\right)$ brane as drawn in the example above. The same is true for the slopes of the branes, since they are related to the charges by supersymmetry. The two $(p, q)$ charges correspond to the values of two central charges of the Fock representation, e.g. the central charges of $\mathcal{F}_{q, t^{-1}}^{(r, s)}$ are $\left((t / q)^{\frac{r}{2}},(t / q)^{\frac{s}{2}}\right)$. The two central charges are denoted by $\left(\gamma, \sqrt{\psi_{0}^{+} / \psi_{0}^{-}}\right)$in appendix A of [7]. See [25-32] for some applications of the algebraic formalism to 5 -brane networks.

The deformation parameters $q$ and $t^{-1}$ of the DIM algebra are interpreted as $\Omega$ background parameters of the theory. To incorporate the permutation symmetry between $q, t^{-1}$ and $t / q$ one has to assume that in addition to the $\mathbb{R}^{2}$ plane of the picture there are three more complex dimensions in the background. We also know that the Fock representation $\mathcal{F}_{q, t^{-1}}^{(0,1)}$ is the representation of the algebra on the equivariant $K$-theory of the Hilbert scheme of points on $\mathbb{C}^{2}$, which corresponds to a $5 d \mathcal{N}=1$ gauge theory living on $\mathbb{C}_{q} \times \mathbb{C}_{t^{-1}} \times S^{1}$, so the background should also contain an $S^{1}$. Thus, Type IIB theory lives on $\mathbb{C}_{q} \times \mathbb{C}_{t^{-1}} \times \mathbb{C}_{t / q} \times \mathbb{R}_{\text {picture }}^{2} \times \mathbb{R} \times S^{1}$. Using the mnemonic from appendix A.5 of [7] the representations of the DIM algebra can then be assigned to branes living in this background and fixed by the equivariant $\mathrm{U}(1)^{2}$ action on $\mathbb{C}^{3}$. The dictionary is summarized in the table below: ${ }^{4}$

\begin{tabular}{|c|c|c|c|c|c|c|c|}
\hline Brane & DIM rep & $\mathbb{C}_{q}$ & $\mathbb{C}_{t^{-1}}$ & $\mathbb{C}_{t / q}$ & $\begin{array}{ll}\text { picture } \\
\mathbb{R} & \mathbb{R} \\
\end{array}$ & $\begin{array}{c}t \\
\mathbb{R}_{t}\end{array}$ & $S^{1}$ \\
\hline F1 & generators & & & & - & & - \\
\hline D1 & generators & & & & - & & - \\
\hline $\mathrm{D} 5_{q, t^{-1}}$ & $\mathcal{F}_{q, t^{-1}}^{(0,1)}$ & -- & -- & & - & & - \\
\hline $\mathrm{D} 5_{q, t / q}$ & & -- & & -- & - & & - \\
\hline $\mathrm{D} 5_{t^{-1}, t / q}$ & $\mathcal{F}_{t^{-1, t / q}}^{(0,1)}$ & & -- & -- & - & & - \\
\hline $\mathrm{NS} 5_{q, t^{-1}}$ & $\mathcal{F}_{q, t^{-1}}^{(1,0)}$ & -- & -- & & - & & - \\
\hline $\mathrm{NS} 5_{q, t / q}$ & $\mathcal{F}_{q, t / q}^{(1,0)}$ & -- & & -- & - & & - \\
\hline $\mathrm{NS}_{t^{-1}, t / q}$ & $\mathcal{F}_{t^{-1, t / q}}^{(1,0)}$ & & -- & -- & - & & - \\
\hline $\mathrm{D} 3_{q}$ & $\mathcal{V}_{q}$ & -- & & & & - & - \\
\hline $\mathrm{D} 3_{t^{-1}}$ & $\mathcal{V}_{t^{-1}}$ & & -- & & & - & - \\
\hline $\mathrm{D} 3_{t / q}$ & $\mathcal{V}_{t / q}$ & & & -- & & - & - \\
\hline $\mathrm{D} 7-\overline{\mathrm{D} 7} ?$ & $\mathcal{M}^{(0, c)}$ & -- & -- & -- & & $|-|$ & - \\
\hline
\end{tabular}

The first two lines in the table (1.3) are the examples of $(p, q)$-strings, namely the fundamental string and the D1 brane. These will play the role of the generators of the

\footnotetext{
${ }^{4}$ Changing $\mathbb{C}_{q} \times \mathbb{C}_{t^{-1}} \times \mathbb{C}_{t / q}$ to $\left(\widetilde{\mathbb{C}^{2} / \mathbb{Z}_{N}}\right)_{q, t^{-1}} \times \mathbb{C}_{t / q}$ corresponds to considering the algebra $U_{q, t}\left(\widehat{\widehat{\mathfrak{g l}}}{ }_{N}\right)$ instead of $U_{q, t}\left(\widehat{\mathfrak{g l}}_{1}\right)$, see [13].
} 
algebra. More precisely DIM algebra generators will live at points where $(p, q)$ strings attach to other branes. We will comment on this in section 3.

As we have already seen, 5-branes spanning different directions are associated with different types of Fock representations. They are depicted by lines (and entries in (1.3)) of different colors as in [7]: $\left(q, t^{-1}\right) 5$-branes are violet, $(q, t / q)$ ones are red and $\left(t^{-1}, t / q\right)$ are blue. Notice that the triple junction (1.2) is allowed only when all three 5-branes are of the same color. The spectral parameter $u$ of the Fock representation $\mathcal{F}^{(r, s)}(u)$ corresponds to the position of the brane in the plane of the picture (complexified by adding the Wilson line along $S^{1}$ of the gauge field on the brane worldvolume).

The additional ingredient introduced in [7] were the D3 branes corresponding to three types of the so-called vector representations $\mathcal{V}_{q}, \mathcal{V}_{t^{-1}}$ and $\mathcal{V}_{t / q}$. Again, the branes are assigned colors according to complex planes they span. The representation $\mathcal{V}_{q}$ of DIM algebra is a representation by difference operators $x$ and $p=q^{x \partial_{x}}$ on functions of a single variable. ${ }^{5}$ This representation is invariant under the $\operatorname{SL}(2, \mathbb{Z})$ automorphism group, as is the D3 brane of Type IIB string theory.

Classically (with equivarant deformation turned off, $q \rightarrow 1$ ), the variables $x$ and $p$ are (the complexifications of) the coordinates in the plane of the picture $\mathbb{R}_{\text {picture }}^{2}$ which plays the role of the phase space. In the quantum regime the D3 branes are delocalized in $\mathbb{R}_{\text {picture }}^{2}$ and only their wavefunctions, defined on the Lagrangian submanifolds are meaningful. The dashed lines featuring in [7] are such wavefunctions with fixed horizontal position: a state in the vector representation $\mathcal{V}_{q}$ with fixed $x=w$ is denoted by a vertical dashed line with spectral parameter $w$ :

$$
\begin{gathered}
\mathcal{V}_{q} \\
w \\
w \\
\vdots \\
\vdots
\end{gathered}=|w\rangle=\delta(x / w)
$$

The higgsed vertices $\Phi$ introduced in [7] are the junctions between NS5 branes and D3 branes of various kinds. For geometric reasons only junctions in which the NS5 and D3 branes share a $\mathbb{C}$ plane are allowed. In our convention this means that the colors of dashed and solid lines in the junction cannot coincide. An example of a D3-NS5 junction is the operator $\Phi_{q, t^{-1}}^{q}: \mathcal{V}_{q} \otimes \mathcal{F}_{q, t^{-1}}^{(1,0)}(u) \rightarrow \mathcal{F}_{q, t^{-1}}^{(1,0)}(t u)$ :

$$
\Phi_{q, t^{-1}}^{q}(|w\rangle \otimes \ldots)=\underline{t u}
$$

where we have already plugged in the state $|w\rangle \in \mathcal{V}_{q}$ into the intertwiner. There is naturally, a dual intertwiner $\Phi_{q}^{* q, t^{-1}}: \mathcal{F}_{q, t^{-1}}^{(1,0)}(u) \rightarrow \mathcal{F}_{q, t^{-1}}^{(1,0)}\left(t^{-1} u\right) \otimes \mathcal{V}_{q}$. One can find explicit expressions for the intertwiners $\Phi$ and $\Phi^{*}$ in [7].

Let us briefly comment on the last row in the table (1.3). Its interpretation is not completely clear. The MacMahon representation $\mathcal{M}^{(0, c)}$ of DIM algebra is the representation on plane partitions (or $3 d$ Young diagrams) and allows for an arbitrary value of the central

\footnotetext{
${ }^{5}$ In [7] these representations had an additional spectral parameter, e.g. $\mathcal{V}_{q}(w)$. However, a more natural way to understand $w$ is as a parameter of a state inside the representation, the representation being independent of $w$.
} 
charge $c . \mathcal{M}^{(0, c)}$ can be built by tensoring an infinite number of Fock spaces with specific spectral parameters and then taking an irreducible part of the product [33]. It turns out that for $c$ tuned to certain quantized values only a finite number of Fock spaces are needed to build $\mathcal{M}^{(0, c)}$. It is therefore natural to assume that this representation should be

1. related to the equivariant $K$-theory of the Hilbert scheme of points on $\mathbb{C}^{3}$ : the basis in the $K$-theory is labelled by plane partitions, which also constitute a basis in $\mathcal{M}^{(0, c)}$ for generic $c$.

2. for specific values of $c$ it should contain irreducible subrepresentations corresponding to stacks of 5-branes. Indeed, in [24] it was shown that for $c=n \ln q-m \ln t$ with $n, m \in \mathbb{N}$ the Macmahon representation $\mathcal{M}^{(0, c)}$ is reducible, while its irreducible subquotient $\mathcal{N}^{(n, m)}$ is a representation spanned by plane partitions with a "pit", i.e. a forbidden box at $(n+1, m+1,1)$. According to [24] the subquotient representation $\mathcal{N}^{(n, m)}$ can alternatively be constructed as a quotient of a tensor product of $(n+m)$ Fock representations corresponding to a stacks of $n \mathrm{D} 5_{t^{-1}, t / q}$ and $m \mathrm{D} 5_{q, t / q}$ branes. The tentative brane interpretation of the two constructions of $\mathcal{N}^{(n, m)}$ is that a D7 brane and an anti-D7 brane partially annihilate leaving behind the stacks of D5 branes. The picture with stacks of D5-branes spanning various $\mathbb{C}$-planes inside $\mathbb{C}_{q, t^{-1}, t / q}^{3}$ is related by $T$-dualities to stacks of $\mathrm{D} 3$ branes stretched on a triple junction of 5 -branes considered in [17-23].

This leads us to believe that the relevant system is a pair of D7 and anti-D7 brane sitting $c$ distance apart in the plane of the picture. However, such configuration seems to break all supersymmetry making this interpretation doubtful. We will not focus on the MacMahon representation here and only briefly mention it in section 4.8 .

In this short note we extend the dictionary proposed in [7] in several directions. In particular we propose:

1. Intertwiners corresponding to junctions of D5 branes with D3 branes. They correspond to pictures such as

$$
\tilde{\Phi}_{q, t^{-1}}^{* q}=u
$$

These operators can be described very explicitly using Young diagrams.

2. Brane crossings for a pair 5-branes and a 5-brane crossing 3-brane. An example of a crossing between a D5 and an NS5 branes is

$$
X_{q, t^{-1}}^{q, t^{-1}}=\frac{\left.\sqrt{\frac{q}{t} u}\right|^{\downarrow} u}{\downarrow \sqrt{\frac{t}{q}} w}
$$

The crossings turn out to have a clear algebraic meaning: they are Lax matrices satisfying the Yang-Baxter relations with the DIM $R$-matrix [34-36]. 
Employing these new ingredients we show how D3 branes arise from 5-brane configurations for quantized values of the spectral parameters. We also clarify the relation between $S$-duality of $\mathcal{N}=43 d$ theories and the $\operatorname{SL}(2, \mathbb{Z})$ automorphism group of the quantum toroidal algebra $U_{q, t}\left(\widehat{\widehat{\mathfrak{g l}}}_{1}\right)$.

The plan of the paper is as follows. In section 2 we introduce new types of intertwiners corresponding to a D3 brane meeting a D5 brane. In section 3 we consider the action of the algebra itself as an intertwining operator and related it to $(p, q)$-string junctions. In section 4 we introduce various brane crossings and clarify their algebraic meaning. In section 5 we establish the precise relation between the networks involving only the conventional refined topological vertices and those with higgsed vertices. In section 6 using the new formalism we consider the brane construction of $3 d T[\mathrm{SU}(N)]$ theory and its cousins and analyze their $S$-duality properties. We present our conclusions in section 7 .

\section{D3 brane joins D5 brane}

In [7] we have introduced the D3-NS5 junctions such as (1.5). Here we would like to add the D3-D5 junctions to our vocabulary. In our pictorial notation these junctions are between a (vertical) dashed line corresponding to vector representation e.g. $\mathcal{V}_{q}$ meeting a vertical solid line corresponding to a Fock representation. As in the case of D3-NS5 junctions, the merging branes should share $\mathbb{C}_{q}$ part of their worldvolume so D $3_{q}$ brane can meet only $\mathrm{D} 5_{q, t^{-1}}$ and $\mathrm{D} 5_{q, t / q}$ corresponding to the Fock representations $\mathcal{F}_{q, t^{-1}}^{(0,1)}$ and $\mathcal{F}_{q, t / q}^{(0,1)}$ respectively. We denote the corresponding intertwiners by $\tilde{\Phi}_{q, t^{-1}}^{* q}$ and $\tilde{\Phi}_{q, t / q}^{* q}$ respectively and draw them as follows:

$$
\tilde{\Phi}_{q, t^{-1}}^{* q}=\tilde{\Phi}_{q, t / q}^{\mathcal{F}_{q, t^{-1}}^{(0,1)}(u)}=\mathcal{F}_{\mathcal{V}_{q} \otimes \mathcal{F}_{q, t^{-1}}^{(0,1)}\left(t^{-1} u\right)}
$$

Notice the shift of the spectral parameter of the Fock representation after the junction. This shift is the same as in the D3-NS5 junctions $\Phi_{q, t^{-1}}^{* q}$ and $\Phi_{q, t / q}^{* q}$ mentioned after eq. (1.5).

One can deduce the explicit expression for the D3-D5 intertwiners from the semiinfinite construction of the vertical Fock representations of DIM algebra [33]. The vertical Fock representation $\mathcal{F}_{q, t^{-1}}^{(0,1)}(u)$ can be understood as a (appropriately regularized) limit of an infinite tensor product of certain subspaces of the vector representations $\mathcal{V}_{q}$. In particular a state in the standard basis ${ }^{6}|\lambda, u\rangle \in \mathcal{F}_{q, t^{-1}}^{(0,1)}(u)$ parametrized by a Young diagram $\lambda=\left[\lambda_{1}, \lambda_{2}, \ldots\right]$ is obtained from the product

$$
|\lambda, u\rangle_{\mathcal{F}_{q, t^{-1}}^{(0,1)}(u)}=\lim _{N \rightarrow \infty}^{\prime}\left|u q^{\lambda_{1}}\right\rangle_{\mathcal{V}_{q}} \otimes\left|u q^{\lambda_{2}} t^{-1}\right\rangle_{\mathcal{V}_{q}} \otimes\left|u q^{\lambda_{3}} t^{-2}\right\rangle_{\mathcal{V}_{q}} \otimes \ldots \otimes\left|u t^{-N+1}\right\rangle_{\mathcal{V}_{q}}
$$

\footnotetext{
${ }^{6}$ This is usually taken to be the basis of Macodnald polynomials however this will not be important for us.
} 
where the prime denotes the regularization. Taking the first factor from the tensor product and treating it as an independent vector representation ${ }^{7}$ one arrives at the formula for the intertwiner

$$
\tilde{\Phi}_{q, t^{-1}}^{* q}|\lambda, u\rangle_{\mathcal{F}_{q, t^{-1}}^{(0,1)}(u)}=\left|q^{\lambda_{1}} u\right\rangle_{\mathcal{V}_{q}} \otimes\left|\lambda \backslash \lambda_{1}, t^{-1} u\right\rangle_{\mathcal{F}_{q, t^{-1}}^{(0,1)}\left(t^{-1} u\right)}
$$

where $\lambda \backslash \lambda_{1}$ denotes the Young diagram $\left[\lambda_{2}, \lambda_{3}, \ldots\right]$. The formulas for $\tilde{\Phi}_{q, t / q}^{* q}$ and for all other allowed combinations of Fock and vector spaces are obtained by permuting $\left(q, t^{-1}, t / q\right)$.

Using the explicit formulas for the action of DIM algebra generators on the vertical Fock (see e.g. [12]) and vector representation (see appendix A.4 of [7]) one can directly verify that the operator defined by (2.3) indeed satisfies the intertwining relations

$$
\tilde{\Phi}_{q, t^{-1}}^{* q} g=\Delta(g) \tilde{\Phi}_{q, t^{-1}}^{* q},
$$

where $\Delta$ is the "vertical" DIM coproduct (see A.2 of [7]). The explicit checks of eq. (2.4) are given in appendix A. From the symmetry between $\left(q, t^{-1}, t / q\right)$ we find that all junctions of this type are given by the formulas similar to (2.3).

\section{$3(p, q)$-string joins D3 brane: the brane nature of DIM representations}

In this section we briefly explain how representations of DIM algebra arise from junctions of $(p, q)$ strings with a given brane. The type of the brane naturally determines the representation of the algebra as summarized in table (1.3). We will focus of the simplest example of the $\mathrm{D} 3_{q}$ brane corresponding to the vector representation $\mathcal{V}_{q}$. The action of DIM algebra currents $x^{ \pm}(z), \psi^{ \pm}(z)$ in this representation are given by

$$
\begin{aligned}
x^{+}(z)|w\rangle_{\mathcal{V}_{q}} & =-\frac{1}{1-q^{-1}} \delta\left(\frac{w}{z}\right)|q w\rangle_{\mathcal{V}_{q}}, \\
x^{-}(z)|w\rangle_{\mathcal{V}_{q}} & =-\frac{1}{1-q} \delta\left(\frac{w}{q z}\right)\left|\frac{w}{q}\right\rangle_{\mathcal{V}_{q}}, \\
\psi^{+}(z)|w\rangle_{\mathcal{V}_{q}} & =\frac{\left(1-\frac{t}{q} \frac{w}{z}\right)\left(1-\frac{1}{t} \frac{w}{z}\right)}{\left(1-\frac{w}{z}\right)\left(1-\frac{1}{q} \frac{w}{z}\right)}|w\rangle_{\mathcal{V}_{q}} \\
\psi^{-}(z)|w\rangle_{\mathcal{V}_{q}} & =\frac{\left(1-\frac{q}{t} \frac{z}{w}\right)\left(1-t \frac{z}{w}\right)}{\left(1-\frac{z}{w}\right)\left(1-q \frac{z}{w}\right)}|w\rangle_{\mathcal{V}_{q}}
\end{aligned}
$$

DIM currents can be expanded as series in $z$ with coefficients $P_{(r, s)}$ :

$$
\begin{aligned}
x^{ \pm}(z) & =\sum_{n \in \mathbb{Z}} P_{(n, \pm 1)} q^{\frac{ \pm 1-n}{2}} z^{-n}, \\
\psi^{ \pm}(z) & =\exp \left[\sum_{n \geq 1} \frac{\kappa_{n}}{n} P_{( \pm n, 0)} q^{\mp \frac{n}{2}} z^{\mp n}\right] .
\end{aligned}
$$

\footnotetext{
${ }^{7}$ This argument is similar to the Hilbert's Grand Hotel paradox: if a guest in the first room of a fully occupied infinite hotel moves out, the hotel manager can still retain full occupancy by moving every guest from room $i$ to room $i-1$.
} 
The generators $P_{(r, s)}$ corresponds to a junction of an $(r, s)$-string with the D3 brane. We draw $(r, s)$-string as a black wavy line in the plane of the picture at slope $(r, s)$ :

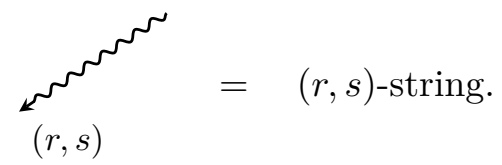

The string is colorless for the following reason. Recall from table (1.3) that $(r, s)$-strings don't span any $\mathbb{C}$-planes on which the equivariant group acts, and therefore remain invariant under the permutation of $\left(q, t^{-1}, t / q\right)$. Indeed, this coincides with what we know about the DIM algebra: it is symmetric under such permutations.

The junction of a $(0,1)$-string with a $\mathrm{D} 3_{q}$ brane corresponds to the following relation following from (3.1):

$$
P_{(0,1)}|w\rangle_{\mathcal{V}_{q}}=-\frac{1}{q^{1 / 2}-q^{-1 / 2}}|q w\rangle_{\mathcal{V}_{q}}
$$

Indeed, using the wavy line eq. (3.8) can be drawn as follows:

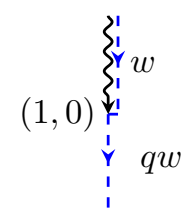

The junction changes the position of the D3 brane by $q$ in the same way as the D3 brane joining D5 brane changes its spectral parameter in eq. (2.1). Similarly we can draw the action of a $(0,-1)$-string:

$$
P_{(0,-1)}|w\rangle_{\mathcal{V}_{q}}=-\frac{1}{q^{-1 / 2}-q^{1 / 2}}\left|\frac{w}{q}\right\rangle_{\mathcal{V}_{q}} \quad \Leftrightarrow \quad \frac{w}{q} \prod^{\frac{1}{1}}(0,-1)
$$

Fundamental strings don't change the position of the D3 brane:

$$
P_{(1,0)}|w\rangle_{\mathcal{V}_{q}}=-\frac{w}{q^{-1 / 2}-q^{1 / 2}}|w\rangle_{\mathcal{V}_{q}} \quad \Leftrightarrow \quad \underset{\vdots}{\vdots} \begin{gathered}
\vdots \\
\vdots \\
\vdots \\
\vdots
\end{gathered}
$$

Similarly we could have drawn pictures for other more complicated representations associated with 5 -branes. However, in that case the $(p, q)$-string junction would not change the position of the brane, but only alter the state (the Young diagram) living on it.

Let us comment on the 5-brane case, which is fundamental for the $(r, s)$-string interpretation of the generators $P_{(r, s)}$. The concrete formulas for the action of $P_{r, s}$ on the 5-branes are obtained from the Fock representation of $U_{q, t^{-1}}\left(\widehat{\hat{\mathfrak{g l}}}_{1}\right)$ (see e.g. [12]). The states $|\lambda\rangle$ of the Fock representation are identified with (equivariant $K$-theoretic classes of the moduli spaces of) U(1) instantons on $\mathbb{C}^{2}$. For example the generators $P_{(0,1)}\left(\right.$ resp. $\left.P_{(0,-1)}\right)$ add (resp. remove) an instanton, taking $|\lambda\rangle$ into a superposition of $|\lambda+\square\rangle$ (resp. $|\lambda-\square\rangle$ ). In this way the quantum toroidal algebra generated by $P_{(r, s)}$ acts on the equivariant $K$-theory by Nakajima correspondences [1-3]. This corroborates our identification of $P_{r, s}$ as $(r, s)$-strings, since $\mathrm{U}(1)$ instantons can be viewed as bound states of D1 branes with a D5 brane. 


\section{5-brane crossings}

The next new element we are going to introduce is what we call the 5-brane crossings, though the branes really cross only in the projection to the plane of the picture, $\mathbb{R}_{\text {picture }}^{2}$. In reality the operators we are going to introduce correspond to the skew branes: pairs of lines approaching each other in a $3 d$ space but failing to intersect.

In section 1 we have summarized that 5 -branes of the same color from table (1.3) spanning lines in the plane of the picture $\mathbb{R}_{\text {picture }}^{2}$ can join into a brane web and that this web corresponds to a network of intertwiners of Fock representations of DIM algebra. However, in this construction we have implicitly assumed that the 5-branes lie at the same value of $t$ on the $\mathbb{R}_{t}$ line - otherwise they have no chance to meet.

One can wonder what algebraic object corresponds to a $\mathrm{D} 5_{q, t^{-1}}$ and an $\mathrm{NS} 5_{q, t^{-1}}$ branes lying at different values of $t$. Two such branes lie along two skew lines in the $3 d$ space $\mathbb{R}_{\text {picture }}^{2} \times \mathbb{R}_{t}$ never touching each other, so one might think that the corresponding operator should be trivial. This intuition turns out to be correct for $q=t$, however, in general it fails and the brane crossing is a nontrivial operator on the tensor product of two Fock spaces which we denote by $X_{q, t^{-1}}^{q, t^{-1}}$. In fact there are two distinct situations: either NS5 brane lies above D 5 brane or below it (we imagine positive $t$ direction goes upwards):

$$
X_{q, t^{-1}}^{q, t^{-1}}(u, w)=\frac{\downarrow_{u}^{w}}{\downarrow} \longleftarrow \quad \bar{X}_{q, t^{-1}}^{q, t^{-1}}(u, w)=\left.\longleftarrow\right|^{w} u
$$

In field theory terms the pictures (4.1) correspond to a Higgs branch of a $5 d$ gauge theory engineered by the 5-brane web (one could assume that the elementary crossings (4.1) are incorporated into a larger brane web). In this case the separation of the branes in the $t$ direction correspond to moving along the Higgs branch.

\subsection{5-brane crossing arise from degenerate resolved picture}

Both of the situations depicted in (4.1) can be continuously deformed into the picture where the two branes lie in the same plane of constant $t$ and do touch each other. When this happens one can "resolve" the crossing as follows:

$$
C(u, w, Q)=\mathcal{F}_{q, t^{-1}}^{(1,0)}(u / Q) \longrightarrow \mathcal{F}_{q, t^{-1}}^{(0,1)}(Q w)
$$

The resolution is parametrized by a complex number $Q$. The first important assumption in our search for the operators $X_{q, t^{-1}}^{q, t^{-1}}$ and $\bar{X}_{q, t^{-1}}^{q, t^{-1}}$ corresponding to (4.1) is that they are obtained from the resolved crossing $C(u, w, Q)$ by specializing to certain particular values of $Q$. In other words, we assume that when $Q$ is tuned to these special values the branes 
are allowed to detach from each other out of the constant $t$ slice of the $3 d$ space and when this happens the operators don't change any further. In particular, they are independent of the separation of the branes along $\mathbb{R}_{t}$. They do, however, depend on the ordering along this direction, so that the two operators $X_{q, t^{-1}}^{q, t^{-1}}$ and $\bar{X}_{q, t^{-1}}^{q, t^{-1}}$ in (4.1) are distinct.

\subsection{5-brane crossing is the DIM Lax operator}

The second key observation from which we deduce the crossing operators is that when the branes are separated in the $t$ direction the corresponding operators must satisfy the Yang-Baxter equation. This property follows from the corresponding equality in the braid group, which in turn appears when we look at the movement of the branes (lines) in the $3 d$ space $\mathbb{R}_{t} \times \mathbb{R}_{\text {picture. }}$ The branes are points in the plane perpendicular to the lines and the topology of their configuration space is described by the braid group.

We already know from [35] that the operators $C(Q)$ satisfy the Yang-Baxter equation with the DIM $R$-matrix up to a function of spectral parameters, which we call an anomaly. However, for two special values of $Q_{ \pm}=(q / t)^{ \pm \frac{1}{2}}$ the anomaly vanishes, so the operators $C\left((q / t)^{ \pm \frac{1}{2}}\right)$ do satisfy the Yang-Baxter equation exactly. They are therefore specializations of the universal DIM $R$-matrix $\mathcal{R}$ in a tensor product of a vertical and horizontal Fock representations. Moreover, these two operators are inverses of each other. We therefore postulate that

$$
\begin{aligned}
& X_{q, t^{-1}}^{q, t^{-1}}(u, w)=C\left(u, w,(t / q)^{\frac{1}{2}}\right)=\left.\mathcal{R}\right|_{\mathcal{F}_{q, t^{-1}}^{(0,1)}(w) \otimes \mathcal{F}_{q, t^{-1}}^{(1,0)}(u)}, \\
& \bar{X}_{q, t^{-1}}^{q, t^{-1}}(u, w)=C\left(u, w,(q / t)^{\frac{1}{2}}\right)=\left.\mathcal{R}^{-1}\right|_{\mathcal{F}_{q, t^{-1}}^{(0,1)}(w) \otimes \mathcal{F}_{q, t^{-1}}^{(1,0)}(u)},
\end{aligned}
$$

In other words, the crossing operators are Lax operators of DIM algebra, satisfying the $R L L$ relations.

Let us give some explicit formulas for the crossing operator. We do this in two different ways and demonstrate that the answers match:

1. In section 4.2.1 we combine explicit expressions for the 5-brane junctions $\Psi_{q, t^{-1}}$ from eq. (1.2) and $\Psi_{q, t^{-1}}^{*}$.

2. In section 4.2.2 we evaluate the universal DIM $R$-matrix $\mathcal{R}$ in the tensor product of the vertical and horizontal Fock representations.

\subsubsection{Lax operator from two 5-brane junctions}

For simplicity we will limit ourselves to the matrix elements of the crossing operators between the vacuum states in the vertical Fock space, which we call $\chi_{q, t^{-1}}^{q, t^{-1}}(u, w)$ and $\bar{\chi}_{q, t^{-1}}^{q, t^{-1}}(u, w)$ :

$$
\chi_{q, t^{-1}}^{q, t^{-1}}(u, w)=(\ldots \otimes\langle\varnothing, \sqrt{t / q} w|) X_{q, t^{-1}}^{q, t^{-1}}(u, w)(|\varnothing, w\rangle \otimes \ldots)=\frac{|\varnothing, w\rangle}{\downarrow}, \leftarrow
$$




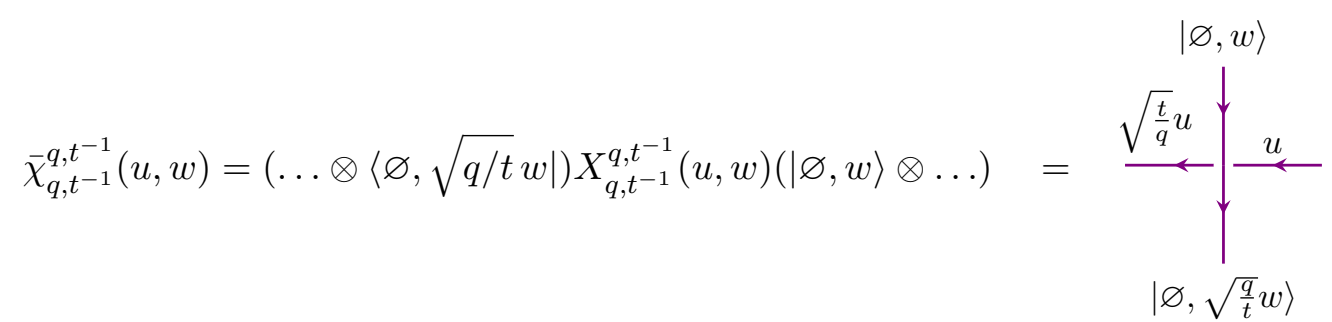

$\chi_{q, t^{-1}}^{q, t^{-1}}(u, w)$ and $\bar{\chi}_{q, t^{-1}}^{q, t^{-1}}(u, w)$ are vertex operators acting on the horizontal Fock space. To calculate them we use explicit expressions for the 5-brane junctions with vacuum state on one of the legs [12]:

$$
\begin{gathered}
\Psi_{q, t^{-1}}(|\varnothing, w\rangle \otimes \ldots)=(-w)^{Q}: \exp \left[\sum_{n \neq 0} \frac{w^{-n}}{n} \frac{a_{n}}{1-q^{-n}}\right]: \\
\left(\ldots \otimes\left\langle\varnothing,(t / q)^{ \pm \frac{1}{2}} w\right|\right) \Psi_{q, t^{-1}}^{*}=(-w)^{-Q}: \exp \left[-\sum_{n \neq 0} \frac{w^{-n}}{n}\left(\frac{t}{q}\right)^{\frac{|n| \mp n}{2}} \frac{a_{n}}{1-q^{-n}}\right]:
\end{gathered}
$$

where $a_{n}$ form a Heisenberg algebra acting on the horizontal Fock space:

$$
\left[a_{n}, a_{m}\right]=n \frac{1-q^{|n|}}{1-t^{|n|}} \delta_{n+m, 0},
$$

and $Q$ is the operator shifting the spectral parameter of the Fock space:

$$
(-w)^{Q}|u, \lambda\rangle=|-w u, \lambda\rangle .
$$

Combining the intertwiners as in the picture (4.2) we get the expressions for the crossing operators:

$$
\begin{aligned}
& \chi_{q, t^{-1}}^{q, t^{-1}}(u, w)=(q ; q, t)_{\infty}\left(\frac{q}{t}\right)^{\frac{Q}{2}} \exp \left[-\sum_{n \geq 1} \frac{w^{n}}{n} \frac{\left(1-(t / q)^{n}\right)}{1-q^{n}} a_{-n}\right], \\
& \bar{\chi}_{q, t^{-1}}^{q, t^{-1}}(u, w)=(t ; q, t)_{\infty}\left(\frac{t}{q}\right)^{\frac{Q}{2}} \exp \left[\sum_{n \geq 1} \frac{w^{-n}}{n} \frac{\left(1-(t / q)^{n}\right)}{1-q^{-n}} a_{n}\right],
\end{aligned}
$$

where

$$
(x ; q, t)_{\infty}=\prod_{i, j \geq 0}\left(1-q^{i} t^{j} x\right) .
$$

The numerical prefactors in eqs. (4.10) are inessential since they are independent of the brane positions and we drop them henceforth for brevity.

\subsubsection{Lax operator from the universal DIM $R$-matrix}

First of all, we observe that there are shifts in spectral parameters of both lines participating the crossing:

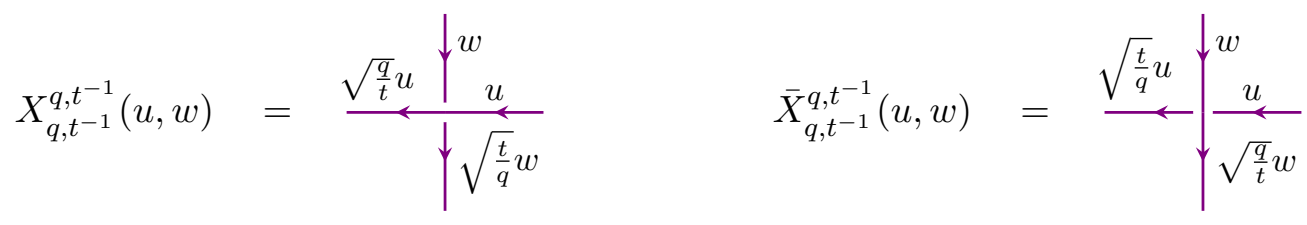


These shifts match with the structure of the universal $R$-matrix, which can be written as a product of four parts (see $[37,38])$ :

$$
\mathcal{R}=P \mathcal{R}_{0} \mathcal{R}_{1} \mathcal{R}_{2},
$$

where $P$ is the permutation operator for the two tensor factors and

$$
\mathcal{R}_{0}=e^{-c \otimes d-d \otimes c-c_{\perp} \otimes d_{\perp}-d_{\perp} \otimes c_{\perp}},
$$

where $c, c_{\perp}$ and $d, d_{\perp}$ are the central charges and the grading operators of the DIM algebra respectively. The $\mathcal{R}_{1}$ and $\mathcal{R}_{2}$ parts of the $R$-matrix have Khoroshkin-Tolstoy form ${ }^{8}$ [40, 41]:

$$
\begin{aligned}
& \mathcal{R}_{1}=\exp \left[-\sum_{n \geq 1} \frac{n}{\kappa_{n}} \gamma^{-\frac{n}{2}} H_{n}^{+} \otimes \gamma^{\frac{n}{2}} H_{-n}^{-}\right] \\
& \mathcal{R}_{2}=\prod_{s=\frac{a}{b} \in(0, \infty)}^{\curvearrowleft} \mathcal{R}_{s}=1+\kappa_{1} \sum_{i \in \mathbb{Z}} x_{i}^{+} \otimes x_{-i}^{-}+\ldots
\end{aligned}
$$

where $\kappa_{n}=\left(1-q^{n}\right)\left(1-t^{-n}\right)\left(1-(t / q)^{n}\right)$, the definitions of $x_{i}^{ \pm}$are given in appendix A of [7] and the generators $H_{n}^{ \pm}$are related to $\psi^{ \pm}(z)$ of [7] by

$$
\psi^{ \pm}(z)=\psi_{0}^{ \pm} \exp \left[\sum_{n \geq 1} H_{ \pm n}^{ \pm} z^{\mp n}\right] .
$$

The element $\mathcal{R}_{2}$ is given by an infinite product of terms, whose explicit form is given e.g. in [37]. What will be important for us is that there is that $\mathcal{R}_{2}$ can be written as a graded expansion in the grading counted by $d_{\perp}$. This expansion starts as in eq. (4.16) with $x_{i}^{+}$ (resp. $x_{-i}^{-}$) having grading 1 (resp. $(-1)$ ).

The part which produces the shifts of the spectral parameters in (4.12) is $\mathcal{R}_{0}$. To see this we notice that the central charges of the horizontal Fock representation are

$$
\left.\left(e^{c}, e^{c_{\perp}}\right)\right|_{\mathcal{F}_{q, t^{-1}}^{(1,0)}(u)}=\left(\sqrt{\frac{t}{q}}, 1\right),
$$

while the vertical representation is obtained by acting with $\operatorname{SL}(2, \mathbb{Z})$ transformation $\left(\begin{array}{cc}0 & 1 \\ -1 & 0\end{array}\right)$ on eq. (4.18):

$$
\left.\left(e^{c}, e^{c_{\perp}}\right)\right|_{\mathcal{F}_{q, t^{-1}}^{(0,1)}(u)}=\left(1, \sqrt{\frac{q}{t}}\right) .
$$

The grading operator $d$ acts on the horizontal Fock representation as a box-counting operator:

$$
d|\lambda, u\rangle_{\mathcal{F}_{q, t^{-1}}^{(1,0)}(u)}=|\lambda||\lambda, u\rangle_{\mathcal{F}_{q, t^{-1}}^{(1,0)}(u)},
$$

\footnotetext{
${ }^{8}$ The sign in the exponent in the $\mathcal{R}_{1}$ part is different in [38] and [39]. We use that of [38]. We also use the modified definitions of $H_{n}^{ \pm}$compared to [38, 39]: $\left(H_{n}^{ \pm}\right)_{\text {our }}=\gamma^{\frac{n}{2}}\left(H_{n}^{ \pm}\right)_{[38,39]}$, corresponding to $\left(\psi^{ \pm}(z)\right)_{\text {our }}=\left(\psi^{ \pm}\left(\gamma^{-1 / 2} z\right)\right)_{[38,39]}$.
} 
while $d_{\perp}$ is strictly speaking not defined as an operator on the horizontal Fock space. It shifts the spectral parameter $u$ of the representation:

$$
Q^{d_{\perp}}|\lambda, u\rangle_{\mathcal{F}_{q, t^{-1}}^{(1,0)}(u)}=|\lambda, Q u\rangle_{\mathcal{F}_{q, t^{-1}}^{(1,0)}(Q u)^{-}}
$$

Therefore, the operator $e^{-c_{\perp} \otimes d_{\perp}}$ acts by shifting the spectral parameter of the horizontal Fock representation by the value of the central charge of the vertical Fock representation. Combining all grading operators together we get

$\mathcal{R}_{0}|\mu, w\rangle_{\mathcal{F}_{q, t^{-1}}^{(0,1)}(w)} \otimes|\lambda, u\rangle_{\mathcal{F}_{q, t^{-1}}^{(1,0)}(u)}=\left(\frac{t}{q}\right)^{\frac{|\lambda|-|\mu|}{2}}\left|\mu, \sqrt{\frac{t}{q} w}\right\rangle_{\mathcal{F}_{q, t^{-1}}^{(0,1)}\left(\sqrt{\frac{t}{q}} w\right)} \otimes\left|\sqrt{\frac{q}{t}} \lambda, u\right\rangle_{\mathcal{F}_{q, t^{-1}}^{(1,0)}\left(\sqrt{\frac{q}{t}} u\right)}$.

This is the pattern of the spectral parameter shifts drawn in (4.12). It can also be written using the momentum shift operator $Q$ as in eq. (4.10).

As in section 4.2.1 we are interested in vacuum matrix elements (4.4) of the Lax operator. These matrix elements can be calculated explicitly from the universal $R$-matrix since the part $\mathcal{R}_{2}$ of the $R$-matrix (4.13) does not contribute. This happens because all elements of negative $d_{\perp}$ grading annihilate the vertical ket vacuum $|\varnothing, w\rangle$ and those of positive grading annihilate the bra vacuum $\left\langle\varnothing,(q / t)^{ \pm 1 / 2} w\right|$.

The remaining $\mathcal{R}_{1}$ part of the $R$-matrix is also easy to calculate if we recall the expressions for the DIM algebra generators $H_{n}^{ \pm}$for vertical and horizontal Fock representations (see e.g. [12]):

$$
\begin{aligned}
H_{n}^{+}|\varnothing, w\rangle_{\mathcal{F}_{q, t^{-1}}^{(0,1)}(w)} & =\frac{w^{n}}{n}\left(1-(t / q)^{n}\right)|\varnothing, w\rangle_{\mathcal{F}_{q, t^{-1}}^{(0,1)}(w)}, \\
H_{-n}^{-}|\varnothing, w\rangle_{\mathcal{F}_{q, t^{-1}}^{(0,1)}(w)} & =\frac{w^{-n}}{n}\left(1-(q / t)^{n}\right)|\varnothing, w\rangle_{\mathcal{F}_{q, t^{-1}}^{(0,1)}(w)} . \\
\left.H_{n}^{+}\right|_{\mathcal{F}_{q, t^{-1}}^{(1,0)}(u)} & =-\frac{\left(1-t^{n}\right)\left(1-\left(\frac{t}{q}\right)^{n}\right)}{n}\left(\frac{q}{t}\right)^{\frac{n}{4}} a_{n}, \\
\left.H_{-n}^{-}\right|_{\mathcal{F}_{q, t^{-1}}^{(1,0)}(u)} & =\frac{\left(1-t^{-n}\right)\left(1-\left(\frac{t}{q}\right)^{n}\right)}{n}\left(\frac{q}{t}\right)^{\frac{n}{4}} a_{-n},
\end{aligned}
$$

where $a_{n}$ are the Heisenberg generators satisfying (4.8). Therefore

$$
\mathcal{R}_{1}\left(|\varnothing, w\rangle_{\mathcal{F}_{q, t^{-1}}^{(0,1)}(w)} \otimes \ldots\right)=\exp \left[-\sum_{n \geq 1} \frac{w^{n}}{n} \frac{\left(1-(t / q)^{n}\right)}{\left(1-q^{n}\right)} a_{-n}\right]
$$

and

$$
\chi_{q, t^{-1}}^{q, t^{-1}}(u, w)=\left(\frac{q}{t}\right)^{\frac{Q}{2}} \exp \left[-\sum_{n \geq 1} \frac{w^{n}}{n} \frac{\left(1-(t / q)^{n}\right)}{1-q^{n}} a_{-n}\right]
$$

exactly as in eq. (4.10).

To get $\bar{\chi}_{q, t^{-1}}^{q, t^{-1}}(u, w)$ we should compute the inverse of the universal $R$-matrix, for which extra caution is needed because the factors $\mathcal{R}_{0}, \mathcal{R}_{1}$ and $\mathcal{R}_{2}$ do not commute with each 
other. We begin by noting that

$$
\mathcal{R}^{-1}=\mathcal{R}_{2}^{-1} \mathcal{R}_{1}^{-1} \mathcal{R}_{0}^{-1} P=P\left(\mathcal{R}_{2}^{\mathrm{op}}\right)^{-1}\left(\mathcal{R}_{1}^{\mathrm{op}}\right)^{-1}\left(\mathcal{R}_{0}^{\mathrm{op}}\right)^{-1},
$$

where ${ }^{\text {op }}$ denotes the exchange of two factors in the tensor product. The parts which will be relevant for us are $\mathcal{R}_{0}$ and $\mathcal{R}_{1}$, for which we have

$$
\begin{aligned}
\left(\mathcal{R}_{0}^{\mathrm{op}}\right)^{-1} & =e^{c \otimes d+d \otimes c+c_{\perp} \otimes d_{\perp}+d_{\perp} \otimes c_{\perp}}, \\
\left(\mathcal{R}_{1}^{\mathrm{op}}\right)^{-1} & =\exp \left[\sum_{n \geq 1} \frac{n}{\kappa_{n}} \gamma^{\frac{n}{2}} H_{-n}^{-} \otimes \gamma^{-\frac{n}{2}} H_{n}^{+}\right] .
\end{aligned}
$$

We then would like to move $\left(\mathcal{R}_{0}^{\mathrm{op}}\right)^{-1}$ past $\left(\mathcal{R}_{1}^{\mathrm{op}}\right)^{-1}$ to bring the inverse $R$-matrix in the same form as the original one:

$$
\left(\mathcal{R}_{0}^{\mathrm{op}}\right)^{-1}=\left(\mathcal{R}_{0}^{\mathrm{op}}\right)^{-1} \exp \left[\sum_{n \geq 1} \frac{n}{\kappa_{n}} \gamma^{-\frac{n}{2}} H_{-n}^{-} \otimes \gamma^{\frac{n}{2}} H_{n}^{+}\right] .
$$

Since we are computing the vacuum matrix element, the $\mathcal{R}_{2}$ can be ignored, so after substituting $H_{n}^{ \pm}$from eqs. (4.23)- (4.26) we have

$$
\exp \left[\sum_{n \geq 1} \frac{n}{\kappa_{n}} \gamma^{-\frac{n}{2}} H_{-n}^{-} \otimes \gamma^{\frac{n}{2}} H_{n}^{+}\right]\left(|\varnothing, w\rangle_{\mathcal{F}_{q, t^{-1}}^{(0,1)}(w)} \otimes \ldots\right)=\exp \left[\sum_{n \geq 1} \frac{w^{-n}}{n} \frac{\left(1-(t / q)^{n}\right)}{\left(1-q^{-n}\right)} a_{n}\right] .
$$

Combining the pieces of the inverse $R$-matrix together we get

$$
\bar{\chi}_{q, t^{-1}}^{q, t^{-1}}(u, w)=\left(\frac{t}{q}\right)^{\frac{Q}{2}} \exp \left[\sum_{n \geq 1} \frac{w^{-n}}{n} \frac{\left(1-(t / q)^{n}\right)}{1-q^{-n}} a_{n}\right]
$$

We find that the formulas (4.3) indeed match the answers (4.10) obtained from multiplication of a pair of intertwining operators. We have thus verified that our definition of the crossing operator is self-consistent: the degenerate resolution (4.2) with two branes meeting in the same constant $t$ plane indeed reproduces the universal $R$-matrix and hence satisfies the Yang-Baxter equation. Of course, the Yang-Baxter equation for such crossings can also be checked directly as was done in $[34,35]$.

\subsection{Functoriality of the crossing}

Many interesting properties follow from eqs. (4.3). For example, suppose that we have $a$ pair of NS5 branes intersecting a single D5 brane:

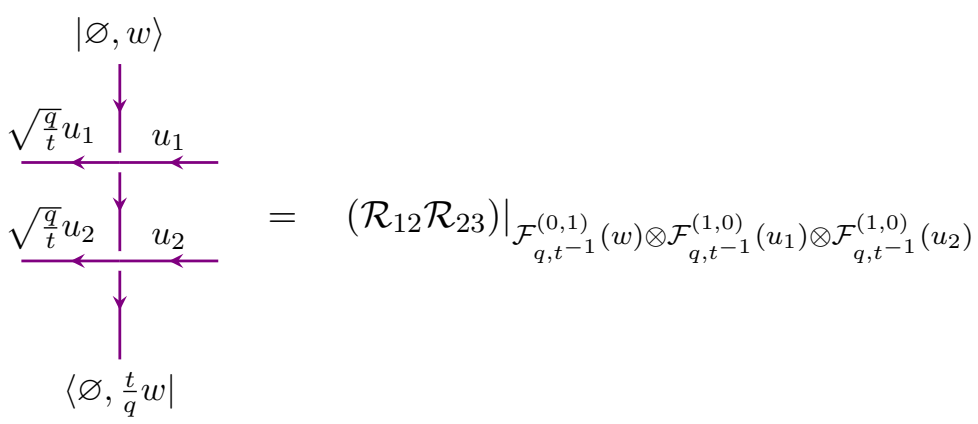


The corresponding combination of operators is a product of two universal $R$-matrices contracted over one of their legs. We can notice that this is precisely the combination appearing in one of the axioms of the universal $R$-matrix:

$$
(\Delta \otimes 1) \mathcal{R}=\mathcal{R}_{12} \mathcal{R}_{23}
$$

Thus, the operator corresponding to the double crossing (4.35) can be found by taking the coproduct of $\mathcal{R}_{0}$ and the generators $H_{-n}^{-}$inside the exponent in $\mathcal{R}_{1}$ (4.15). Notice that the argument from section 4.2.2 about the vanishing of $\mathbb{R}_{2}$ is still valid because it has to do with the vacuum states in the vertical representation on which the coproduct doesn't act. The formulas for the DIM coproduct $\Delta$ can be found in appendix A.2 of [7]. In particular we have

$$
\begin{aligned}
\Delta\left(H_{n}^{ \pm}\right) & =H_{n}^{ \pm} \otimes \gamma^{\mp \frac{n}{2}}+\gamma^{ \pm \frac{n}{2}} \otimes H_{n}^{ \pm}, \\
\Delta(\gamma) & =\gamma \otimes \gamma
\end{aligned}
$$

Applying eqs. (4.37), (4.38) we get:

$$
\begin{aligned}
& \left.\left(\mathcal{R}_{12} \mathcal{R}_{23}\right)\right|_{\mathcal{F}_{q, t^{-1}}^{(0,1)}(w) \otimes \mathcal{F}_{q, t^{-1}}^{(1,0)}\left(u_{1}\right) \otimes \mathcal{F}_{q, t^{-1}}^{(1,0)}\left(u_{2}\right)}=\left.((\Delta \otimes 1) \mathcal{R})\right|_{\mathcal{F}_{q, t^{-1}}^{(0,1)}(w) \otimes \mathcal{F}_{q, t^{-1}}^{(1,0)}\left(u_{1}\right) \otimes \mathcal{F}_{q, t^{-1}}^{(1,0)}\left(u_{2}\right)} \\
& =\left(\frac{q}{t}\right)^{\frac{Q_{1}+Q_{2}}{2}} \exp \left[-\sum_{n \geq 1} \frac{w^{n}}{n} \frac{\left(1-(t / q)^{n}\right)}{1-q^{n}}\left(a_{-n}^{(1)}+\left(\frac{t}{q}\right)^{\frac{n}{2}} a_{-n}^{(2)}\right)\right] \\
& =\chi_{q, t^{-1}}^{q, t^{-1}}\left(u_{1}, w\right) \otimes \chi_{q, t^{-1}}^{q, t^{-1}}\left(u_{2}, \sqrt{\frac{t}{q}} w\right)
\end{aligned}
$$

where $Q_{1,2}$ and $a_{n}^{(1,2)}$ are the shift operators and Heisenberg generators acting on the first and second horizontal Fock spaces respectively. The two factors in the last line of eq. (4.39) are completely factorized. We have thus proven that the double crossing with vacuum states at both ends is just a product of two vacuum crossings:

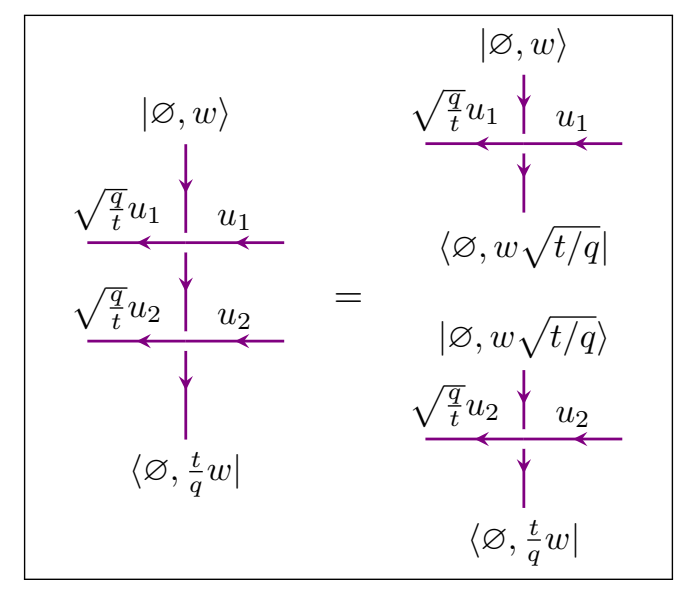

In other words, no nontrivial states are allowed on the segment of the vertical line between the crossings. This phenomenon is in fact well-known in the refined topological vertex 
formalism: degenerate crossings force the vacuum state to "pass through" the crossings downwards. This can also be verified directly using the more direct approach of section 4.2.1.

The factorization argument is general: for any number of horizontal legs crossing a vertical line the resulting operator can be found by taking the coproduct multiple times. The result will be of the form

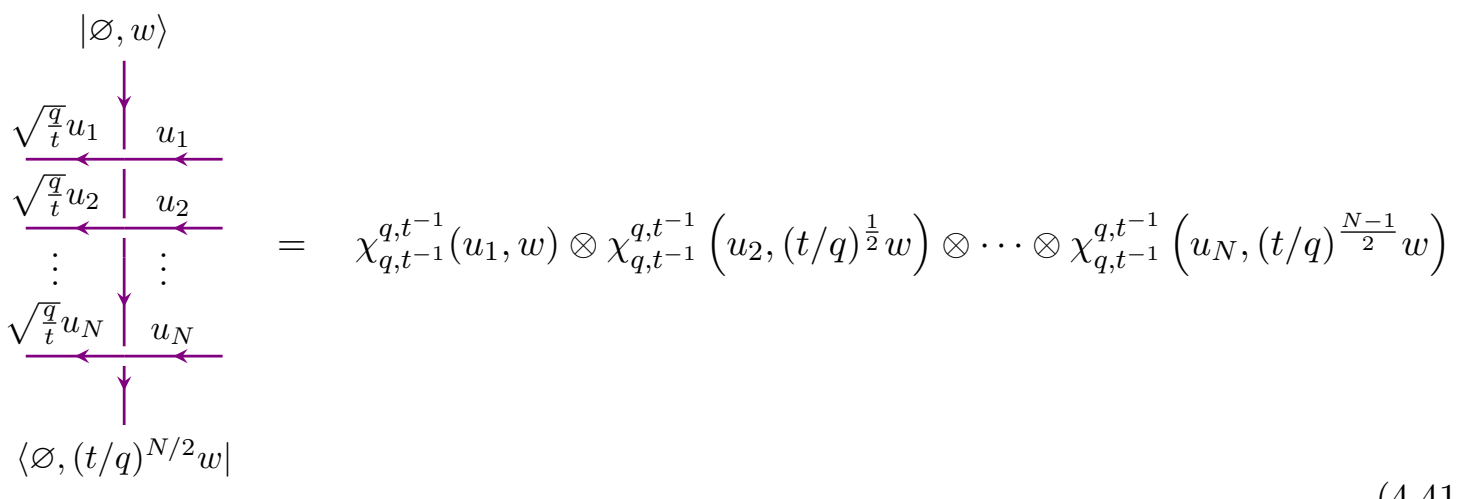

Again, this can also be seen using the refined topological vertices $\Psi$ and $\Psi^{*}$ as in section 4.2.1.

\subsection{Crossing between two 5-branes of different types}

The next logical step in our exploration of the brane-algebra dictionary is to consider a crossing of a D5 and NS5 branes sharing only a single $\mathbb{C}$ factor instead of a $\mathbb{C}^{2}$ plane. Without loss of generality we can consider the crossing between an NS5 $5_{q, t^{-1}}$ brane and a $\mathrm{D} 5_{q, t / q}$ brane:

$$
X_{q, t^{-1}}^{q, t / q}(u, w)=\longleftarrow \uparrow_{\downarrow}^{w} u \quad \bar{X}_{q, t^{-1}}^{q, t / q}(u, w)=\longleftarrow \psi^{w}
$$

Here we don't have the opportunity to deform the picture so that the two branes are brought into the same plane of constant $t$ and merged into a web. However, the crossing should still satisfy the Yang-Baxter equation hence should be a DIM Lax operator. It should act in the tensor product of the vertical Fock space $\mathcal{F}_{q, t / q}^{(0,1)}(w)$ corresponding to D $5_{q, t / q}$ and the horizontal Fock space $\mathbb{F}_{q, t^{-1}}^{(1,0)}(u)$, corresponding to $\mathrm{NS} 5_{q, t^{-1}}$. Therefore, we can write similarly to (4.12) (notice the difference in the indices of the Fock representations):

$$
\begin{aligned}
& X_{q, t^{-1}}^{q, t / q}(u, w)=\left.\mathcal{R}\right|_{\mathcal{F}_{q, t / q}^{(0,1)}(w) \otimes \mathcal{F}_{q, t^{-1}}^{(1,0)}(u)}, \\
& \bar{X}_{q, t^{-1}}^{q, t / q}(u, w)=\left.\mathcal{R}^{-1}\right|_{\mathcal{F}_{q, t / q}^{(0,1)}(w) \otimes \mathcal{F}_{q, t^{-1}}^{(1,0)}(u)} .
\end{aligned}
$$

Using the same arguments as in section 4.2 we can evaluate the vacuum matrix elements of 
the Lax operators (4.43) explicitly:

$$
\begin{aligned}
& \chi_{q, t^{-1}}^{q, t / q}(u, w)=(\ldots \otimes\langle\varnothing, \sqrt{t / q} w|) X_{q, t^{-1}}^{q, t / q}(u, w)(|\varnothing, w\rangle \otimes \ldots)=\frac{\sqrt{t} u \psi_{u}}{\downarrow} \\
& \left|\varnothing, \sqrt{\frac{t}{q}} w\right\rangle \\
& =t^{\frac{Q}{2}} \exp \left[-\sum_{n \geq 1} \frac{w^{n}}{n} \frac{\left(1-t^{-n}\right)}{\left(1-q^{n}\right)} a_{-n}\right], \\
& \bar{\chi}_{q, t^{-1}}^{q, t / q}(u, w)=(\ldots \otimes\langle\varnothing, \sqrt{q / t} w|) X_{q, t^{-1}}^{q, t / q}(u, w)(|\varnothing, w\rangle \otimes \ldots)=\left.\stackrel{u / \sqrt{t}}{\leftarrow}\right|_{\downarrow} ^{|\varnothing, w\rangle} u \\
& =t^{-\frac{Q}{2}} \exp \left[-\sum_{n \geq 1} \frac{w^{-n}}{n}\left(\frac{t}{q}\right)^{n} \frac{\left(1-t^{n}\right)}{\left(1-q^{-n}\right)} a_{n}\right]
\end{aligned}
$$

\subsection{Hanany-Witten D3 brane creation}

There is something curious about the operators (4.44) (4.45), since they very much resemble pieces of the $\mathrm{D} 3_{q^{-}} \mathrm{NS} 5_{q, t^{-1}}$ junction (1.5), which reads [7]

$$
\begin{aligned}
& \Phi_{q, t^{-1}}^{q}(|w\rangle \otimes \ldots)=\quad \begin{array}{r}
w_{\grave{r}}^{\prime} \\
t u
\end{array} \\
& =t^{Q} w^{\frac{\ln u}{\ln q}} \exp \left[-\sum_{n \geq 1} \frac{w^{n}}{n} \frac{1-t^{-n}}{1-q^{n}} a_{-n}\right] \exp \left[\sum_{n \geq 1} \frac{w^{-n}}{n} \frac{1-t^{n}}{1-q^{-n}} a_{n}\right]
\end{aligned}
$$

Comparing eqs. (4.44), (4.45) with eq. (4.46) we arrive at a remarkable formula:

$$
\chi_{q, t^{-1}}^{q, t / q}(u, w)=w^{-\frac{\ln u}{\ln q}+\frac{\ln t}{2 \ln q}} \Phi_{q, t^{-1}}^{q}\left(|w\rangle \otimes \bar{\chi}_{q, t^{-1}}^{q, t / q}\left(u, \frac{t}{q} w\right)\right)
$$

which can be put in pictorial form (we omit the scalar prefactor in the pictures)

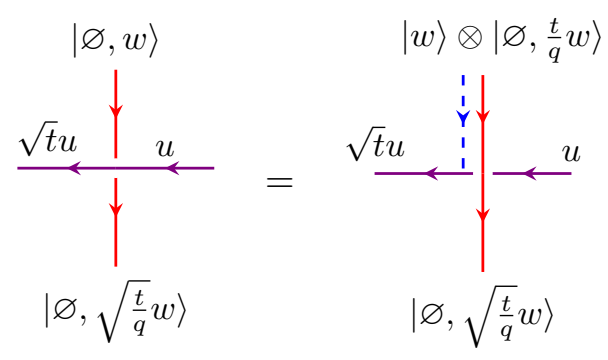


Furthermore, we can make use of the D3-D5 junction (2.3) to write

$$
|w\rangle_{\mathcal{V}_{q}} \otimes\left|\varnothing, \frac{t}{q} w\right\rangle_{\mathcal{F}_{q, t / q}^{(0,1)}(w t / q)}=\tilde{\Phi}_{q, t / q}^{* q}|\varnothing, w\rangle
$$

Eq. (4.49) and the identity (4.47) can be combined compactly into the following picture:

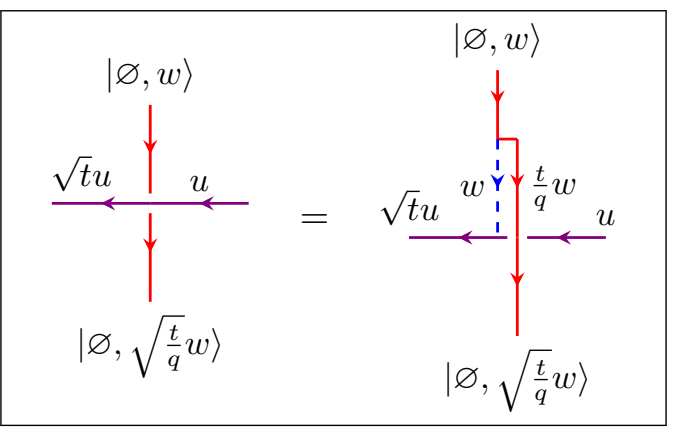

The picture (4.50) is the algebraic incarnation of the Hanany-Witten brane creation effect [42]. When a $\mathrm{D} 5_{q, t / q}$ brane is moved past an $\mathrm{NS} 5_{q, t^{-1}}$ a $\mathrm{D} 3_{q}$ brane is created, which is stretched between the D5 and NS5 branes. This brane move in our language is an equivalence transformation of the network of intertwiners: the networks before and after the move coincide as operators acting between DIM representations. In fact, the relation (4.50) should hold for general states in the vertical Fock representation.

\subsection{Crossing of a NS5 brane and a D3 brane}

Having understood the general pattern - that brane crossings correspond to DIM Lax operators in various representations - we can consider more examples of such operators. Perhaps the simplest example is the D3 brane crossing an NS5 $f_{q, t^{-1}}$ brane. Here there are two possibilities: either the D3 brane is $\mathrm{D} 3_{q}$ or $\mathrm{D} 3_{t^{-1}}$ and shares a $\mathbb{C}$ worldvolume factor with the $\mathrm{NS} 5_{q, t^{-1}}$ brane, or it is the $\mathrm{D} 3_{t / q}$ brane and is completely orthogonal to the $\mathrm{NS}_{q, t^{-1}}$ brane. We consider these possibilities in turn.

\subsection{1 $\mathrm{NS5}_{q, t^{-1}}$ and $\mathrm{D3}_{q}$ branes}

For all the crossings we will be interested in the analogues of the vacuum matrix elements of the corresponding Lax operators. There is no vacuum state in the vector representation, so we are going to use the ket state with fixed vertical position $|w\rangle$ instead of it. The relevant bra state is $\left\langle\sqrt{\frac{t}{q}} w\right|$ because the shift in the spectral parameter of the vertical brane is governed by the central charge of the horizontal brane, which is $\sqrt{\frac{t}{q}}$ in the present case.

We the crossing operators for $\mathrm{NS} 5_{q, t^{-1}}$ and $\mathrm{D} 3_{q}$ branes $x_{q, t^{-1}}^{q}$ and $\bar{x}_{q, t^{-1}}^{q}$ since they are "smaller cousins" of $\chi$ and $\bar{\chi}$ operators from section 4.1. As in section 4.1, 4.2.2 there are two ways to compute the crossings: using a pair of intertwiners $\Phi, \Phi^{*}$, or from the universal $R$-matrix. The same results agree. 
Plugging the expressions for $H_{n}^{ \pm}$in the vector representation $\mathcal{V}_{q}$

$$
\begin{aligned}
& H_{n}^{+}|w\rangle_{\mathcal{V}_{q}}=\frac{w^{n}\left(1-t^{-n}\right)\left(1-\left(\frac{t}{q}\right)^{n}\right)}{n}|w\rangle_{\mathcal{V}_{q}}, \\
& H_{n}^{-}|w\rangle_{\mathcal{V}_{q}}=\frac{w^{-n}\left(1-t^{n}\right)\left(1-\left(\frac{q}{t}\right)^{n}\right)}{n}|w\rangle_{\mathcal{V}_{q}}
\end{aligned}
$$

into the universal $R$-matrix (4.13) we get the crossing operators:

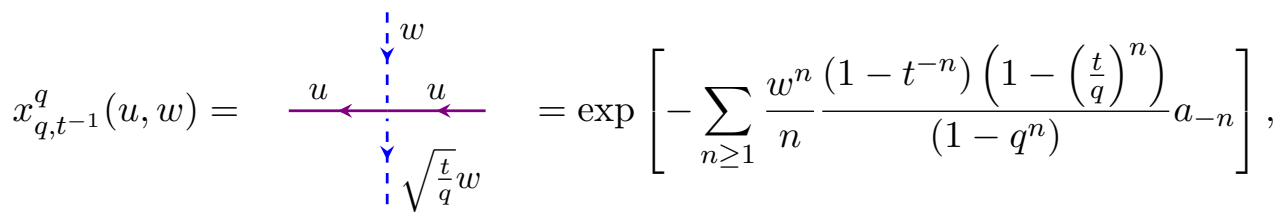

$$
\begin{aligned}
& \bar{x}_{q, t^{-1}}^{q}(u, w)=\stackrel{u}{u} \begin{array}{l}
u \\
\hdashline \sqrt{\frac{q}{t}} w
\end{array}=\exp \left[\sum_{n \geq 1} \frac{w^{-n}}{n} \frac{\left(1-t^{n}\right)\left(1-\left(\frac{t}{q}\right)^{n}\right)}{\left(1-q^{n}\right)} a_{n}\right] .
\end{aligned}
$$

Notice that the spectral parameter of the NS5 brane is unchanged at the crossing with a D3 brane.

\subsection{2 $\mathrm{NS}_{q, t^{-1}}$ and $\mathrm{D} 3_{t / q}$ branes}

In this case the pair of branes shares only the $S^{1}$ direction. As in the previous section we can use the expressions for $H_{n}^{ \pm}$in the vector representation $\mathcal{V}_{t / q}$ (obtained from eqs. (4.51), (4.52) by the exchange $q \leftrightarrow t / q$ ) to get the following crossing operators:

$$
\begin{aligned}
& x_{q, t^{-1}}^{t / q}(u, w)=\frac{u}{\substack{w \\
\hdashline}}=\exp \left[-\sum_{n \geq 1} \frac{w^{n}}{n}\left(1-t^{-n}\right) a_{-n}\right], \\
& \bar{x}_{q, t^{-1}}^{t / q}(u, w)=\frac{u}{y^{\frac{q}{t} w}}=\exp \left[-\sum_{n \geq 1} \frac{w^{-n}}{n}\left(\frac{t}{q}\right)^{n}\left(1-t^{n}\right) a_{n}\right] .
\end{aligned}
$$

\subsection{Hanany-Witten $(p, q)$-string creation}

We notice that the crossing operators (4.55), (4.56) between orthogonal NS5 and D3 branes have particularly simple form. Following the example of section 4.5 we compare $x_{q, t^{-1}}^{t / q}$ with $\bar{x}_{q, t^{-1}}^{t / q}$ and find that they are related using the DIM generating currents $x^{ \pm}(z)$ (see appendix $\mathrm{A}$ of [7] for the complete list of definitions). In the Fock representation $\mathcal{F}_{q, t^{-1}}^{(1,0)}(u)$ 
the generating currents of DIM act as follows:

$$
\begin{aligned}
\left.x^{+}(z)\right|_{\mathcal{F}_{q, t^{-1}}^{(1,0)}(u)}= & \frac{u}{\left(1-q^{-1}\right)(1-t)} \exp \left[\sum_{n \geq 1} \frac{1-t^{-n}}{n} a_{-n} w^{n}\right] \exp \left[-\sum_{n \geq 1} \frac{1-t^{n}}{n} a_{n} w^{-n}\right], \\
\left.x^{-}(z)\right|_{\mathcal{F}_{q, t^{-1}}^{(1,0)}(u)}= & \frac{u^{-1}}{(1-q)\left(1-t^{-1}\right)} \exp \left[-\sum_{n \geq 1} \frac{1-t^{-n}}{n}\left(\frac{t}{q}\right)^{\frac{n}{2}} a_{-n} w^{n}\right] \\
& \times \exp \left[\sum_{n \geq 1} \frac{1-t^{n}}{n}\left(\frac{t}{q}\right)^{\frac{n}{2}} a_{n} w^{-n}\right] .
\end{aligned}
$$

Using the formulas (4.57)-(4.58) we can write the following identity:

$$
\bar{x}_{q, t^{-1}}^{t / q}(u, w)=\left.\frac{\left(1-q^{-1}\right)(1-t)}{u} x_{q, t^{-1}}^{t / q}\left(u, \frac{q}{t} w\right) x^{+}\left(\frac{q}{t} w\right)\right|_{\mathcal{F}_{q, t^{-1}}^{(1,0)}(u)}
$$

Now we notice that the shift in the argument $w \rightarrow \frac{q}{t} w$ in the r.h.s. of eq. (4.59) can be reproduced by the action of $x^{-}(z)$ in the vector representation. More explicitly, the action of generating currents in representation $\mathcal{V}_{t / q}$ reads

$$
\begin{aligned}
& x^{+}(z)|w\rangle_{\mathcal{V}_{t / q}}=-\frac{1}{1-q / t} \delta\left(\frac{w}{z}\right)\left|\frac{t}{q} w\right\rangle_{\mathcal{V}_{t / q}}, \\
& x^{-}(z)|w\rangle_{\mathcal{V}_{t / q}}=-\frac{1}{1-t / q} \delta\left(\frac{q}{t} \frac{w}{z}\right)\left|\frac{q}{t} w\right\rangle_{\mathcal{V}_{t / q}} .
\end{aligned}
$$

Taking into account the delta-function in eq. (4.61) we can write eq. (4.59) in a nice integral form:

$$
\begin{aligned}
\bar{x}_{q, t^{-1}}^{t / q}(u, w)= & -\frac{\left(1-q^{-1}\right)(1-t)(1-t / q)}{u} \\
& \times \oint \frac{d z}{z}(\ldots \otimes\langle\sqrt{t / q} w|) \mathcal{R}\left(\left.x^{-}(z)|w\rangle_{\mathcal{V}_{t / q}} \otimes x^{+}(z)\right|_{\mathcal{F}_{q, t^{-1}}^{(1,0)}(u)}\right) .
\end{aligned}
$$

The identity (4.62) seems complicated but the corresponding picture of intertwiners is a lot more elucidating:

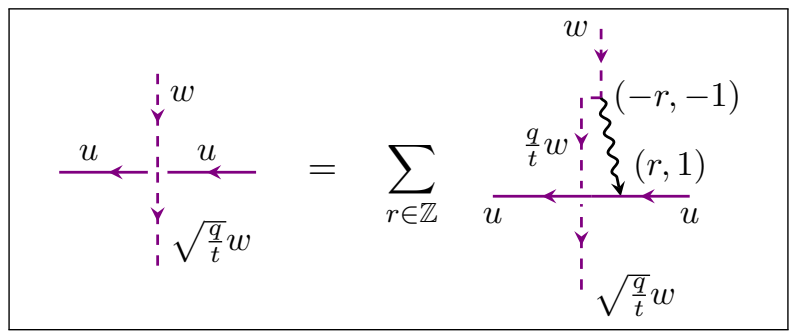

The wavy arrow denotes the $(p, q)$-string, as described in section 3 . The beginning of the arrow puts DIM generator $P_{(-r,-1)}$ on the corresponding brane, while the endpoint of the arrow inserts $P_{(r, 1)}$. The resulting sum over $r$ is the same as the integral over $z$ in eq. (4.62). 
Eq. (4.63) is nothing but another instance of the Hanany-Witten effect: when a D3 brane passes through an orthogonal NS5 brane a $(p, q)$-string is created. But it cannot be just a single $(p, q)$-string for the following reason. The NS5 brane is invariant under a subgroup of the $S$-duality group generated by an element $S T S \in \mathrm{SL}(2, \mathbb{Z})$, and D3 brane is invariant under the whole $\mathrm{SL}(2, \mathbb{Z})$. Therefore the $(p, q)$-brane state should also be invariant, and this is achieved by taking the linear superposition of states with all possible $r$.

\subsection{Crossing of Fock and MacMahon representations}

We can also write the crossing operators between an NS5 brane and whatever brane corresponds to a vertical MacMahon representation $\mathcal{M}^{(0, c)}(w)$. The action of DIM on $\mathcal{M}^{(0, c)}(w)$ is given by [39]:

$$
\begin{aligned}
H_{n}^{+}|\varnothing, w\rangle_{\mathcal{M}^{(0, c)}(w)} & =\frac{w^{n}}{n}\left(1-K^{n}\right)|\varnothing, w\rangle_{\mathcal{M}^{(0, c)}(w)}, \\
H_{-n}^{-}|\varnothing, w\rangle_{\mathcal{M}^{(0, c)}(w)} & =\frac{w^{-n}}{n}\left(1-K^{-n}\right)|\varnothing, w\rangle_{\mathcal{M}^{(0, c)}(w)},
\end{aligned}
$$

where $K=e^{c}$ parametrizes the central charge of the representation. We have

$$
\begin{aligned}
& \frac{|\varnothing, w\rangle}{\Downarrow} \longleftarrow=K^{-Q} \exp \left[-\sum_{n \geq 1} \frac{w^{n}}{n} \frac{\left(1-K^{n}\right)}{\left(1-q^{n}\right)} a_{-n}\right], \\
& \left|\varnothing, \sqrt{\frac{t}{q}} w\right\rangle \\
& |\varnothing, w\rangle \\
& \underline{K u} \longleftarrow u=K^{Q} \exp \left[-\sum_{n \geq 1} \frac{w^{-n}}{n}\left(\frac{t}{q}\right)^{n} \frac{\left(1-K^{-n}\right)}{\left(1-q^{-n}\right)} a_{n}\right], \\
& \left|\varnothing, \sqrt{\frac{q}{t}} w\right\rangle
\end{aligned}
$$

where the double line denotes the MacMahon representation. We have discussed some hints about the role of MacMahon modules in the Introduction, but this part in our brane/algebra dictionary is still not clear.

\section{$5 \quad$ Higgsed vertices from refined topological vertices}

In this section we explain how obtain the higgsed intertwiners of [7] from the intertwiners of [12]. In more physical terms we show how to obtain D3 brane junctions from the junctions involving only 5-branes.

In section 4.1 we have argued that the operator corresponding to crossing $\mathrm{NS} 5_{q, t^{-1}}$ and $\mathrm{D} 5_{q, t^{-1}}$ branes can be obtained from a degenerate resolved picture, where two 5 -brane 
junctions are placed "near each other":

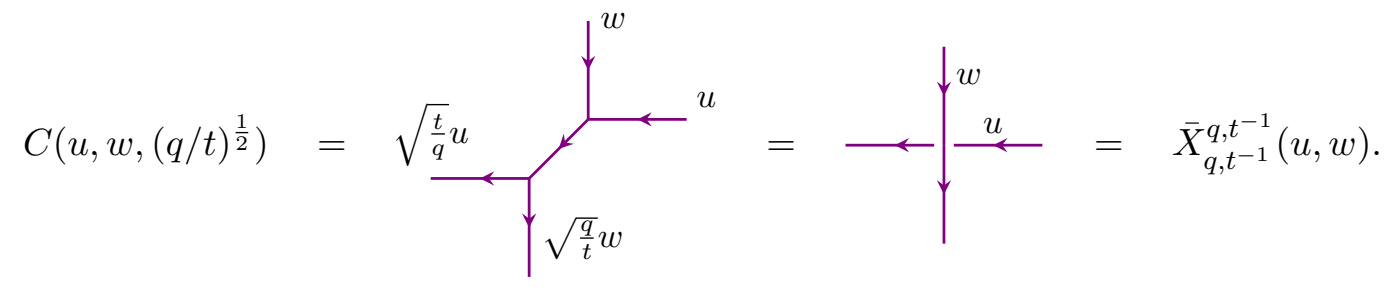

We would like to understand what happens if we tune the parameters of the brane web in the l.h.s. to another value, with the parameter of the resolution being $Q=\sqrt{\frac{t}{q}} t$. Making an explicit computation with intertwining operators $\Psi$ and $\Psi^{*}$ one finds that the resulting operator is a product of two: the crossing $\bar{X}_{q, t^{-1}}^{q, t^{-1}}(u, t w)$ and the higgsed intertwiner $\Phi_{q, t^{-1}}^{q}$ :

$$
\begin{aligned}
& C\left(u, w,(q / t)^{\frac{1}{2}} t^{-1}\right)\left(|\lambda, w\rangle_{\mathcal{F}_{q, t^{-1}}^{(0,1)}(w)} \otimes \ldots\right) \\
& \quad=\Phi_{q, t^{-1}}^{q}\left(\left|q^{\lambda_{1}} w\right\rangle_{\mathcal{V}_{q}} \otimes \bar{X}_{q, t^{-1}}^{q, t^{-1}}\left(u, t^{-1} w\right)\left(\left|\lambda \backslash \lambda_{1}, w\right\rangle_{\mathcal{F}_{q, t^{-1}}^{(0,1)}(w)} \otimes \ldots\right)\right) .
\end{aligned}
$$

We can now use the intertwiner from section 2 to merge the states $\left|q^{\lambda_{1}}\right\rangle$ and $\left|\lambda \backslash \lambda_{1}, w\right\rangle_{\mathcal{F}_{q, t^{-1}}^{(0,1)}(w)}$. We can then draw the following picture of intertwiners:

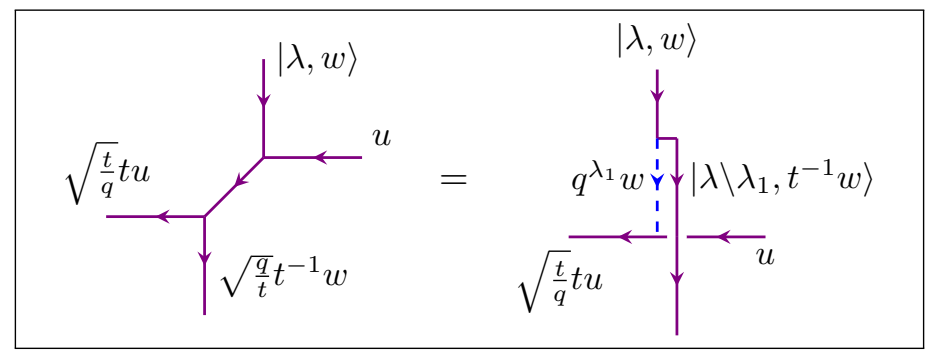

The identity (5.3) is the reason why the higgsing procedure works: one can tune the parameters of the 5-brane web so that a D3 brane appears. Notice that eq. (5.3) identity differs in an essential way from Hanany-Witten identity eq. (4.50), where the 5-branes, being orthogonal to each other, cannot merge. Since (5.3) is an operator identity, one can use it as a part of any networks of intertwiners - the identity applies locally.

One more useful identity when transforming 5-brane webs into higgsed networks is the following brane move, which follows directly from the functoriality of crossing operators:

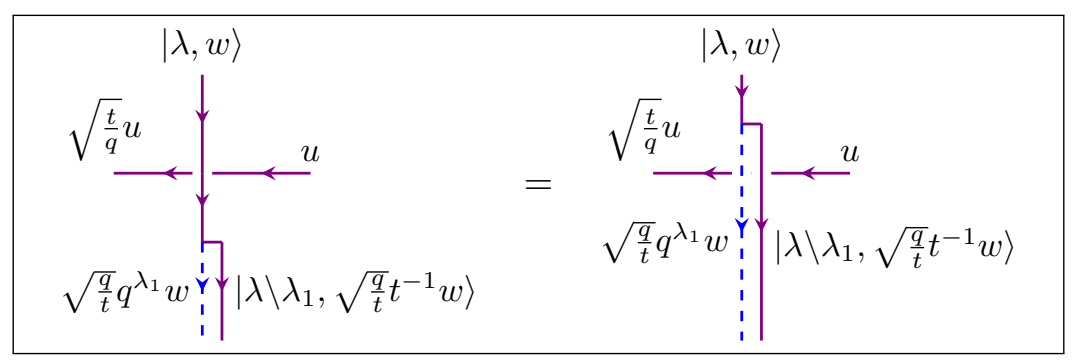

In the next section will see how using the identities (5.3) and (5.4) one can transform a web of 5-branes with tuned parameters into a higgsed network of intertwiners. 


\section{$6 \quad T[\mathrm{SU}(N)]$ vs $\boldsymbol{F T}[\mathrm{SU}(N)]$ partition functions and brane factors}

In this section we will study a nontrivial example of a $3 d$ quiver gauge theory, which can be obtained from our brane constructions. The theory is denoted by $T[\mathrm{SU}(N)]$ it is a linear quiver with gauge groups $\mathrm{U}(1) \times \mathrm{U}(2) \times \ldots \times \mathrm{U}(N-1)$ and a frozen $\mathrm{U}(N)$ flavour symmetry at one end. This theory can be thought of as counting maps from a $3 d$ space into full flag variety $\operatorname{Fl}(1,2,3, \ldots, N)$. The theory has $\mathcal{N}=4$ supersymmetry in flat space, but for nontrivial equivariant parameter $t$ it is softly broken to $\mathcal{N}=2^{*}$. See e.g. [43, 44] for the review of the properties of $T[\mathrm{SU}(N)]$ theory.

The picture of intertwiners corresponding to this theory is (we draw all the examples for $N=2$ only, but our approach is completely general):

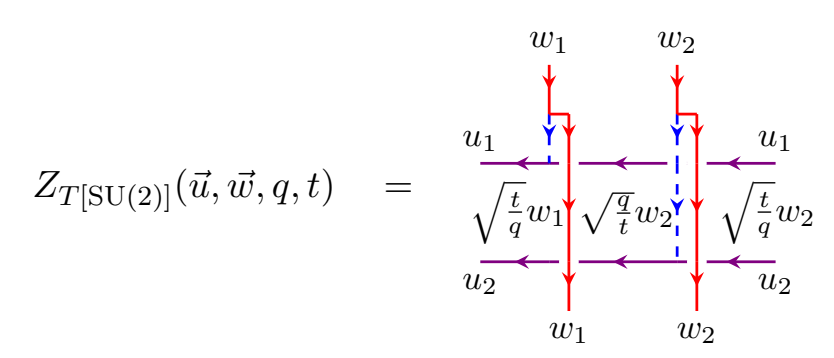

There is a close cousin of the $T[\mathrm{SU}(N)]$ theory called ${ }^{9} F T[\mathrm{SU}(N)]$ for which the red 5-branes in (6.1) are replaced with violet ones:

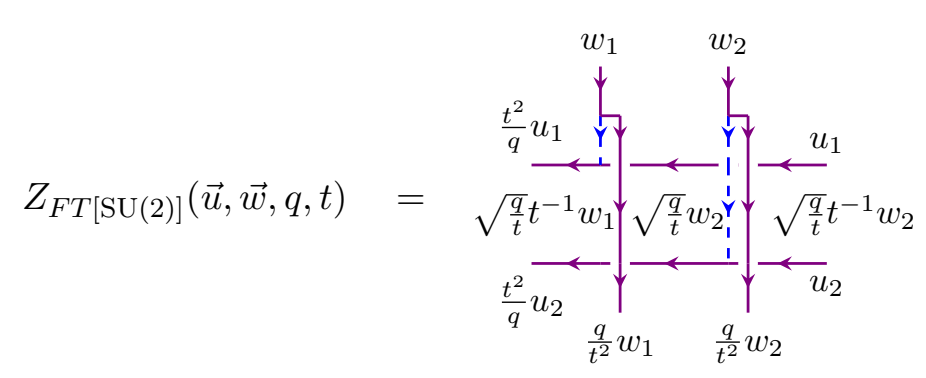

This theory even without equivariant deformation has only $\mathcal{N}=2$ supersymmetry. However, it has almost the same partition function as the $T[\mathrm{SU}(N)]$ theory. Indeed the arrangement of the D3 branes in both theories are the same, and so is the gauge contribution to the partition function. The theories differ only by what is usually called "brane factors" [43, 44] - the contributions of the fields living in the worldvolume of the 5-branes. These fields are non-dynamical from the point of view of the $3 d$ theory and hence give a simple prefactor in front of the partition function. We will explain the origin of the brane factors algebraically in section 6.2.

To do this we employ brane moves on both pictures (6.1), (6.2) first to send the D3-D5 junctions upwards to infinity, so that the D3 and D5 branes can move independently. After that we move D5 branes to the right of the picture, commuting them with all D3 branes that happen on the way. The commutation gives rise to the brane factors. As soon as the D5 branes reach the vacuum state living on the NS5 branes to the right of the picture they disappear. Indeed, $\bar{\chi}$ from eq. (4.10) as well as from eq. (4.45) contain only the annihilation

\footnotetext{
${ }^{9} \mathrm{~F}$ stands for flip of a $\mathbb{C}$ direction spanned by the D5 branes - from $\mathbb{C}_{q, t^{-1}}^{2}$ to $\mathbb{C}_{q, t / q}^{2}$.
} 
operators $a_{n}$ with $n>0$. In this way we are left with the partition function involving only D3 and NS5 branes, which we have already considered in [7].

\subsection{Crossings commute with screening charges}

Consider two NS5 $5_{q, t^{-1}}$ branes with a segment of $\mathrm{D} 3_{q}$ stretched between them. Let there be also a D $5_{q, t^{-1}}$ brane crossing underneath the NS5 branes:

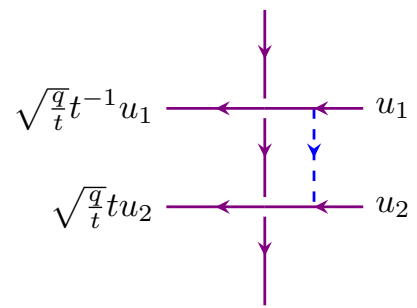

As pointed out in section 4.3, the crossings between the D5 and the NS5 branes are described by the universal $R$-matrix of $U_{q, t}\left(\widehat{\mathfrak{g}}_{1}\right)$ evaluated in tensor products of Fock representations corresponding to the 5-branes. The universal $R$-matrix is an element of the tensor square of the DIM algebra. The second factor of this tensor product acts on the tensor product of two horizontal Fock representations. From this seemingly abstract reasoning one can deduce that the D5 brane commutes with the D3 brane. Indeed, as discussed in detail in [7] the finite D3 brane segment gives the screening charge - the intertwiner of a pair of Fock representations. The screening charge commutes with any element of the DIM algebra, hence with D5 crossing:

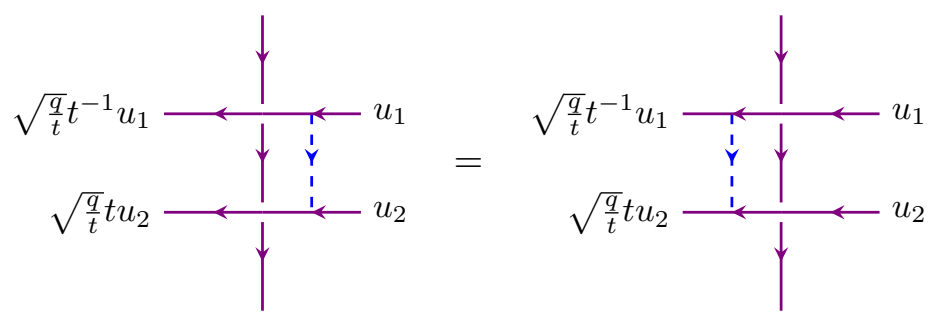

Of course this identity can also be checked by direct computation.

Due to the identity (6.4) when we commute the D5 brane crossings with NS5-D3 junctions, we don't have to worry about the intermediate finite D3 brane segments. Only semi-infinite D3 branes will produce the brane factors.

\subsection{Brane factors from commutation relations between crossings and inter- twiners}

In the previous section we have shown that the screening charges corresponding to finite D3 brane segments commute with D5 branes running beneath or above them. The situation is different for semi-infinite D3 branes - the commutation relations are nontrivial for them. These commutation relations produce the brane factors for the $3 d$ theory. We thus have to take into account only the uppermost NS5 brane on which the seim-infinite parts of D3 branes are located. It is convenient at this point to write the D3-NS5 brane crossings using D3-NS5 brane junctions. 
Using the explicit expressions (4.3), (4.44), (4.45) we calculate the commutators:

$$
\begin{aligned}
\bar{\chi}_{q, t^{-1}}^{q, t^{-1}}(z, u) \Phi_{q, t^{-1}}^{q}|w\rangle \otimes \ldots & =w^{\frac{1-\beta}{2}} \exp \left[\sum_{n \geq 1} \frac{1}{n}\left(\frac{w}{z}\right)^{n} \frac{1-(q / t)^{n}}{1-q^{n}}\right] \Phi_{q, t^{-1}}^{q}|w\rangle \otimes \bar{\chi}_{q, t^{-1}}^{q, t^{-1}}(z, u) \\
& =w^{\frac{1-\beta}{2}} \frac{\left(\frac{q}{t} \frac{w}{z} ; q\right)_{\infty}}{\left(\frac{w}{z} ; q\right)_{\infty}} \Phi_{q, t^{-1}}^{q}|w\rangle \otimes \bar{\chi}_{q, t^{-1}}^{q, t^{-1}}(z, u) . \\
\bar{\chi}_{q, t^{-1}}^{q, t / q}(z, u) \Phi_{q, t^{-1}}^{q}|w\rangle \otimes \ldots & =w^{\frac{\beta}{2}} \exp \left[\sum_{n \geq 1} \frac{1}{n}\left(\frac{w}{z}\right)^{n} \frac{1-t^{n}}{1-q^{n}}\right] \Phi_{q, t^{-1}}^{q}|w\rangle \otimes \bar{\chi}_{q, t^{-1}}^{q, t^{-1}}(z, u) \\
& =w^{\frac{\beta}{2}} \frac{\left(\frac{w}{z} ; q\right)_{\infty}}{\left(t \frac{w}{z} ; q\right)_{\infty}} \Phi_{q, t^{-1}}^{q}|w\rangle \otimes \bar{\chi}_{q, t^{-1}}^{q, t / q}(z, u) .
\end{aligned}
$$

We can then compute the matrix element of several "dressed" intertwiners $\Phi_{q, t^{-1}}^{q}\left(\left|w_{i}\right\rangle \otimes\right.$ $\left.\chi_{q, t^{-1}}^{q, t^{-1}}\left(t^{-1} w_{i}\right)\right)$ (and $\left.\Phi_{q, t^{-1}}^{q}\left(\left|w_{i}\right\rangle \otimes \chi_{q, t^{-1}}^{q, t / q}\left(w_{i} t / q\right)\right)\right)$ in terms of the "undressed" ones $\Phi_{q, t^{-1}}^{q}\left|w_{i}\right\rangle$ :

$$
\begin{aligned}
& \left\langle\varnothing, u_{1}\left|\prod_{i=1}^{n} \Phi_{q, t^{-1}}^{q}\right| w_{i}\right\rangle \otimes \chi_{q, t^{-1}}^{q, t^{-1}}\left(t^{-1} w_{i}\right)\left|\varnothing, u_{1}\right\rangle \\
& =\prod_{i<j}^{n}\left[w_{j}^{\frac{1-\beta}{2}} \frac{\left(q \frac{w_{j}}{w_{i}} ; q\right)_{\infty}}{\left(t \frac{w_{j}}{w_{i}} ; q\right)_{\infty}}\right]\left\langle\varnothing, t^{\frac{3 n}{2}} q^{-\frac{n}{2}} u_{1}\left|\prod_{i=1}^{n} \Phi_{q, t^{-1}}^{q}\right| w_{i}\right\rangle \otimes\left|\varnothing, t^{\frac{n}{2}} q^{-\frac{n}{2}} u_{1}\right\rangle . \\
& \left\langle\varnothing, t^{\frac{n}{2}} u_{1}\left|\prod_{i=1}^{n} \Phi_{q, t^{-1}}^{q}\right| w_{i}\right\rangle \otimes \chi_{q, t^{-1}}^{q, t / q}\left(\frac{t}{q} w_{i}\right)\left|\varnothing, u_{1}\right\rangle \\
& =\prod_{i<j}^{n}\left[w_{j}^{\frac{\beta}{2}} \frac{\left(q \frac{w_{j}}{w_{i}} ; q\right)_{\infty}}{\left(\frac{q}{t} \frac{w_{j}}{w_{i}} ; q\right)_{\infty}}\right]\left\langle\varnothing, t^{\frac{n}{2}} u_{1}\left|\prod_{i=1}^{n} \Phi_{q, t^{-1}}^{q}\right| w_{i}\right\rangle \otimes\left|\varnothing, t^{-\frac{n}{2}} u_{1}\right\rangle .
\end{aligned}
$$

Let us introduce the "undressed" partition function

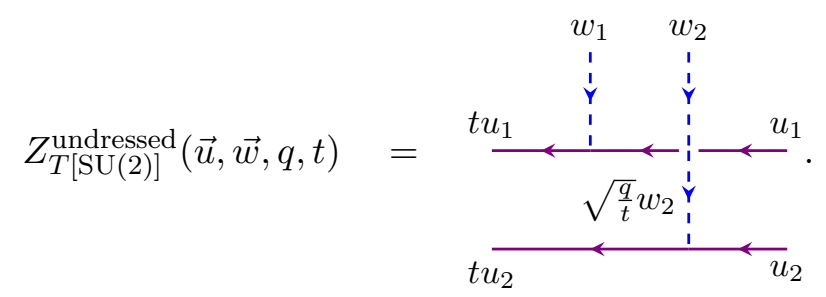

By construction this partition function doesn't feel the difference between the $T[\mathrm{SU}(N)]$ and $F T[\mathrm{SU}(N)]$ theories.

From (6.7) (6.8) we can express two different dressed partition functions through the undressed one and therefore get the relation between the two:

$$
\begin{aligned}
Z_{T[\mathrm{SU}(N)]}(\vec{u}, \vec{w}, q, t) & =\prod_{i<j}^{N}\left[w_{j}^{\frac{\beta}{2}} \frac{\left(q \frac{w_{j}}{w_{i}} ; q\right)_{\infty}}{\left(\frac{q}{t} \frac{w_{j}}{w_{i}} ; q\right)_{\infty}}\right] Z_{T[\operatorname{SU}(2)]}^{\text {undressed }}(\vec{u}, \vec{w}, q, t), \\
Z_{F T[\mathrm{SU}(N)]}(\vec{u}, \vec{w}, q, t) & =\prod_{i<j}^{N}\left[w_{j}^{\frac{1-\beta}{2}} \frac{\left(q \frac{w_{j}}{w_{i}} ; q\right)_{\infty}}{\left(t \frac{w_{j}}{w_{i}} ; q\right)_{\infty}}\right] Z_{T[\operatorname{SU}(2)]}^{\text {undressed }}(\vec{u}, \vec{w}, q, t) .
\end{aligned}
$$


Comparing eqs. (6.10) and (6.11) we finally get the relation between the $T[\mathrm{SU}(N)]$ and $F T[\mathrm{SU}(N)]$ partition functions:

$$
Z_{F T[\mathrm{SU}(N)]}(\vec{u}, \vec{w}, q, t)=\prod_{i<j}^{N}\left[w_{j}^{\frac{1}{2}-2 \beta} \frac{\left(\frac{q}{t} \frac{w_{j}}{w_{i}} ; q\right)_{\infty}}{\left(t \frac{w_{j}}{w_{i}} ; q\right)_{\infty}}\right] Z_{T[\operatorname{SU}(N)]}(\vec{u}, \vec{w}, q, t)
$$

This coincides with the expressions for the flipping fields from e.g. [43, 44].

\section{3 $S$-duality and spectral duality}

$S$-duality of Type IIB theory in our formalism is just the rotation of the picture by ${ }^{10} \frac{\pi}{2}$. In the algebraic language $S$-duality acts on DIM algebra by automorphisms. ${ }^{11}$ Under this rotation the spectral parameters $\vec{u}$ and $\vec{w}$ are exchanged. Using the brane move (5.4) one can bring the $T[\mathrm{SU}(N)]$ brane picture (6.1) into the form symmetric under the mirror image (also under $\frac{\pi}{2}$ rotation, but one needs to commute some branes). However, there is an important caveat that the invariance holds provided one exchanges the red and violet branes after the rotation. This can be achieved by making the substitution $t \leftrightarrow \frac{q}{t}$ : DIM algebra is invariant and the only things that change are the labels of its representations. We therefore have for the $T[\mathrm{SU}(N)]$ partition function:

$$
Z_{T[\mathrm{SU}(N)]}(\vec{u}, \vec{w}, q, t)=Z_{T[\mathrm{SU}(N)]}\left(\vec{w}, \vec{u}, q, \frac{q}{t}\right)
$$

In $F T[\mathrm{SU}(N)]$ theory the situation is different, since one doesn't need to exchange any branes. So, $S$-duality of Type IIB implies:

$$
Z_{F T[\mathrm{SU}(N)]}(\vec{u}, \vec{w}, q, t)=Z_{F T[\mathrm{SU}(N)]}(\vec{w}, \vec{u}, q, t)
$$

This duality can be called spectral, since it is related to the spectral duality in integrable systems. The relation (6.12) together with (6.13) and (6.14) implies a simple transformation rule for $T[\mathrm{SU}(N)]$ under the inversion $t \leftrightarrow \frac{q}{t}$.

\subsection{Dualities in the double elliptic system}

Let us also mention that the diagrams (6.1), (6.2) for $T[\mathrm{SU}(N)]$ and $F T[\mathrm{SU}(N)]$ theories respectively can be compactified in both directions. Naively, this gives rise to a $4 d$ gauge theory with affine quiver. In fact after the compactification the theory on the D3 does not decouple from the sector living on the 5-branes, so the situation is more involved. However, since our brane moves are local we can still apply them to transform the brane diagram as we wish. In this way it is possible to get from our picture a purely 5-brane diagram studied in [45] in the context of double elliptic (Dell) integrable systems.

\footnotetext{
${ }^{10}$ Mirror image along the diagonal is essentially equivalent to the $\frac{\pi}{2}$ rotation in this setup.

${ }^{11} \mathrm{DIM}$ algebra is invariant under the automorphisms, but the coalgebra structure after an $\operatorname{SL}(2, \mathbb{Z})$ transformations is twisted by a nontrivial Drinfeld cocycle. Thus $\operatorname{SL}(2, \mathbb{Z})$ is an automorphism of DIM algebra as a Hopf algebra.
} 
It is remarkable that the duality properties of the compactified partition function can be deduced from the same reasoning as before the compactification. In particular one can compute the brane factors relating the compactified $T[\mathrm{SU}(N)]$ and $F T S U[N]$ theories. They are expressed through double $q$-Pochhammer symbols.

\section{Conclusions and discussions}

In this paper we have enriched the dictionary between Type IIB brane constructions and representations of DIM algebra. We have systematically investigated different types of brane junctions and crossings and found the corresponding intertwining operators. We have argued that the brane crossing operators have a unified algebraic meaning: they are given by the universal $R$-matrix for DIM algebra taken in the representations associated with the pair of crossed branes. We have found remarkable identities between crossing and intertwining operators, which translate the Hanany-Witten brane creation effect into the algebraic language. We have elucidated the higgsing picture, i.e. the identity between 5-brane webs at certain special points of their moduli spaces and brane networks with extra D3 branes. Finally, we have clarified the origin of $S$-duality and spectral duality in $3 d$ gauge theories living on D3 branes stretched between 5-branes.

The structure of the DIM algebra and its representation ring turns out to be so restrictive that it is tempting to propose that Type IIB brane moves can be derived from the DIM algebra. This would mean that the theory (at least after the equivariant deformation) is completely determined by its large hidden symmetry, just as the properties of a $2 d$ CFT can be derived just from knowing the Virasoro algebra.

Though we had some success with the identification of algebraic objects with branes, some points clearly deserve further investigation. In particular, it is still not completely clear what is the role of MacMahon representations. Another crucial point is the coproduct in the DIM algebra whose role in the brane picture remains mysterious. How does the DIM coproduct, which is not invariant under $\mathrm{SL}(2, \mathbb{Z})$ transformations arise from the branes? What is the role of the $R$-matrix in the brane picture? We leave all these questions for the future.

In general, it would be also interesting to investigate the connection between the universal $R$-matrices and the $R$-matrices obtained from the tetrahedron equations as in [51]. Their forms are quite different: the former is the product over positive roots of a root system, while the latter is a trace over an auxiliary space. Nevertheless, they carry the same amount of information at least in the $A_{N}$ case. It would also be interesting to obtain a solution of the tetrahedron equation from which the DIM algebra $R$-matrix can be obtained possibly along the lines of [52].

As shown in [7], our approach reproduces the matrix models computing holomorphic blocks of $3 d$ supersymmetric gauge theories. The brane pictures and rules we have considered here and in [7] can be understood from the purely matrix model point of view. To this end one needs to "project" the pictures on the $\mathbb{R}_{t}$. The D3 brane worldvolumes then become segments separated by points, which represent 5-branes. The integration over the positions of the intermediate D3 branes produces the matrix model. This approach has been pursued 
e.g. in [46], where the action of $\operatorname{SL}(2, \mathbb{Z})$ and some brane rules for the matrix model were introduced. Our $\mathrm{SL}(2, \mathbb{Z})$ action on the DIM representation and intertwiners matches that of [46], however we skip the details not to further overcomplicate our presentation here.

Perhaps one could better understand the brane model we have introduced using the $4 d$ Chern-Simons theory [47-50] with DIM algebra playing the role of the gauge group.

\section{Acknowledgments}

The author thanks M. Bershtein, M. Aganagic and C. Schmid for discussions. The author is supported by the RSF grant 18-71-10073.

\section{A Intertwining property of the D3-D5 junction}

In this appendix we prove the intertwining property (2.4) of the operator $\tilde{\Phi}_{q, t^{-1}}^{* q}$. It is enough to check it for the generating currents $\psi^{ \pm}(z)$ and $x^{ \pm}(z)$, which we undertake in turn. In the process we employ the formulas for the action of $U_{q, t}\left(\widehat{\hat{\mathfrak{g l}}}_{1}\right)$ on $\mathcal{F}_{q, t^{-1}}^{(0,1)}(u)$ from [12].

1. $\psi^{ \pm}(z)$. In the 1.h.s. of eq. (2.4) we have

$$
\begin{aligned}
& \tilde{\Phi}_{q, t^{-1}}^{* q} \psi^{ \pm}(z)|\lambda, u\rangle_{\mathcal{F}_{q, t^{-1}}^{(0,1)}}= \\
= & \left(\frac{q}{t}\right)^{ \pm \frac{1}{2}} \exp \left[\sum_{n \geq 1} \frac{1}{n}\left(\frac{u}{z}\right)^{ \pm n}\left(1-(t / q)^{ \pm n}\right)\left(1-t^{\mp n}\right) \sum_{i \geq 1} q^{ \pm n \lambda_{i}} t^{ \pm n(1-i)}\right] \tilde{\Phi}_{q, t^{-1}}^{* q}|\lambda, u\rangle_{\mathcal{F}_{q, t^{-1}}^{(0,1)}},
\end{aligned}
$$

while on the r.h.s. we use the DIM coproduct from appendix A.2 from [7] and eqs. (3.3), (3.4) (notice that for both representations $\gamma=1$ )

$$
\begin{aligned}
& \Delta\left(\psi^{ \pm}(z)\right)\left|q^{\lambda_{1}} u\right\rangle_{\mathcal{V}_{q}} \otimes\left|\lambda \backslash \lambda_{1}, t^{-1} u\right\rangle_{\mathcal{F}_{q, t^{-1}}^{(0,1)}\left(t^{-1} u\right)} \\
& =\left(\psi^{ \pm}(z) \otimes \psi^{ \pm}(z)\right)\left|q^{\lambda_{1}} u\right\rangle_{\mathcal{V}_{q}} \otimes\left|\lambda \backslash \lambda_{1}, t^{-1} u\right\rangle_{\mathcal{F}_{\mathcal{F}^{(0,1} t^{-1}}^{\left(t^{-1} u\right)}} \\
& =\exp \left[\sum_{n \geq 1} \frac{1}{n}\left(\frac{u}{z}\right)^{ \pm n}\left(1-(t / q)^{ \pm n}\right)\left(1-t^{\mp n}\right)\right] \\
& \times\left(\frac{q}{t}\right)^{ \pm \frac{1}{2}} \exp \left[\sum_{n \geq 1} \frac{1}{n}\left(\frac{u}{z}\right)^{ \pm n}\left(1-(t / q)^{ \pm n}\right)\left(1-t^{\mp n}\right) \sum_{i \geq 1} t^{\mp n} q^{ \pm n \lambda_{i+1}} t^{ \pm n(1-i)}\right] \\
& \times\left|q^{\lambda_{1}} u\right\rangle_{\mathcal{V}_{q}} \otimes\left|\lambda \backslash \lambda_{1}, t^{-1} u\right\rangle_{\mathcal{F}_{q, t^{-1}}^{(0,1)}\left(t^{-1} u\right)} .
\end{aligned}
$$

We observe that eqs. (A.1) and (A.2) are identical, hence, eq. (2.4) holds for $g=\psi^{ \pm}(z)$.

2. $x^{+}(z)$. The 1.h.s. of eq. (2.4) for $g=x^{+}(z)$ reads

$$
\tilde{\Phi}_{q, t^{-1}}^{* q} x^{+}(z)|\lambda, u\rangle_{\mathcal{F}_{q, t^{-1}}^{(0,1)}}=\sum_{i=1}^{l(\lambda)+1} \delta\left(q^{\lambda_{i}} t^{1-i} \frac{u}{z}\right) A_{\lambda, i}^{+} \tilde{\Phi}_{q, t^{-1}}^{* q}\left|u, \lambda+\square_{i}\right\rangle,
$$


where

$$
A_{\lambda, i}^{+}=\left.(1-t) \prod_{j=1}^{i-1} \frac{\left(1-t \frac{y_{i}}{y_{j}}\right)\left(1-\frac{q}{t} \frac{y_{i}}{y_{j}}\right)}{\left(1-\frac{y_{i}}{y_{j}}\right)\left(1-q \frac{y_{i}}{y_{j}}\right)}\right|_{y_{k}=q^{\lambda} k t^{1-k}} .
$$

In the r.h.s. of eq. (2.4) we use the coproduct from appendix A.2 from [7] and eq. (3.1)

$$
\begin{aligned}
& \Delta\left(x^{+}(z)\right)\left|q^{\lambda_{1}} u\right\rangle_{\mathcal{V}_{q}} \otimes\left|\lambda \backslash \lambda_{1}, t^{-1} u\right\rangle_{\mathcal{F}_{q, t^{-1}}^{(0,1)}\left(t^{-1} u\right)} \\
& =\left(x^{+}(z) \otimes 1+\psi^{-}(z) \otimes x^{+}(z)\right)\left|q^{\lambda_{1}} u\right\rangle_{\mathcal{V}_{q}} \otimes\left|\lambda \backslash \lambda_{1}, t^{-1} u\right\rangle_{\mathcal{F}_{q, t^{-1}}^{(0,1)}\left(t^{-1} u\right)} \\
& =-\frac{1}{1-q^{-1}} \delta\left(q^{\lambda_{1}} \frac{u}{z}\right)\left|q^{\lambda_{1}+1} u\right\rangle_{\mathcal{V}_{q}} \otimes\left|\lambda \backslash \lambda_{1}, t^{-1} u\right\rangle_{\mathcal{F}_{q, t^{-1}}^{(0,1)}\left(t^{-1} u\right)} \\
& +\frac{\left(1-t \frac{z}{q^{\lambda_{1} u}}\right)\left(1-\frac{q}{t} \frac{z}{q^{\lambda_{1} u}}\right)}{\left(1-\frac{z}{q^{\lambda_{1} u}}\right)\left(1-q \frac{z}{q^{\lambda_{1} u}}\right)}\left|q^{\lambda_{1}} u\right\rangle_{\mathcal{V}_{q}} \otimes \sum_{i=1}^{l(\lambda)} \delta\left(q^{\lambda_{i+1}} t^{1-i} \frac{t^{-1} u}{z}\right) A_{\lambda \backslash \lambda_{1}, i}\left|\lambda \backslash \lambda_{1}+\square_{i}, t^{-1} u\right\rangle_{\mathcal{F}_{q, t^{-1}}^{(0,1)}\left(t^{-1} u\right)}
\end{aligned}
$$

For eqs. (A.3) and (A.5) coincide it is crucial that $\left[\lambda_{1}, \lambda \backslash \lambda_{1}+\square_{i}\right]$ is indeed a Young diagram. The only case when this might not be true is when $\lambda_{1}=\lambda_{2}$ and $i=1$. However, in this case the delta function corresponding to $i=1$ in the last line of eq. (A.5) cancels with the rational prefactor, so the troublesome term does not arise. In all other cases the terms in eq. (A.3) and (A.5) are in one-to-one correspondence and the coefficients also match, so eq. (2.4) is valid for $x^{+}(z)$.

3. $x^{-}(z)$. The 1.h.s. of eq. (2.4) reads:

$$
\tilde{\Phi}_{q, t^{-1}}^{* q} x^{-}(z)|\lambda, u\rangle_{\mathcal{F}_{q, t^{-1}}^{(0,1)}}=\sum_{i=1}^{l(\lambda)} \delta\left(q^{\lambda_{i}-1} t^{1-i} \frac{u}{z}\right) A_{\lambda, i}^{-} \tilde{\Phi}_{q, t^{-1}}^{* q}\left|u, \lambda-\square_{i}\right\rangle,
$$

where

$$
A_{\lambda, i}^{-}=\left.\left(1-t^{-1}\right) \prod_{j \geq i+1} \frac{\left(1-t^{-1} \frac{y_{j}}{y_{i}}\right)\left(1-\frac{t}{q} \frac{y_{j}}{y_{i}}\right)}{\left(1-\frac{y_{j}}{y_{i}}\right)\left(1-q^{-1} \frac{y_{j}}{y_{i}}\right)}\right|_{y_{k}=q^{\lambda_{k}-\delta_{k, i}} t^{1-k}} .
$$

The r.h.s. of the intertwining relation (2.4) is given by

$$
\begin{aligned}
& \Delta\left(x^{-}(z)\right)\left|q^{\lambda_{1}} u\right\rangle_{\mathcal{V}_{q}} \otimes\left|\lambda \backslash \lambda_{1}, t^{-1} u\right\rangle_{\mathcal{F}_{q, t^{-1}}^{(0,1)}\left(t^{-1} u\right)} \\
& =\left(x^{-}(z) \otimes \psi^{+}(z)+1 \otimes x^{-}(z)\right)\left|q^{\lambda_{1}} u\right\rangle_{\mathcal{V}_{q}} \otimes\left|\lambda \backslash \lambda_{1}, t^{-1} u\right\rangle_{\mathcal{F}_{\mathcal{F}^{\left(0, t^{-1}\right.}}^{(0,1)}\left(t^{-1} u\right)} \\
& =-\frac{1}{1-q} \delta\left(q^{\lambda_{1}-1} \frac{u}{z}\right)\left|q^{\lambda_{1}-1} u\right\rangle_{\mathcal{V}_{q}} \\
& \otimes \sqrt{\frac{q}{t}} \exp \left[\sum_{n \geq 1} \frac{1}{n}\left(\frac{u}{z}\right)^{n}\left(1-(t / q)^{n}\right)\left(1-t^{-n}\right) \sum_{i \geq 1} t^{-n} q^{n \lambda_{i+1}} t^{n(1-i)}\right]\left|\lambda \backslash \lambda_{1}, t^{-1} u\right\rangle_{\mathcal{F}_{q, t^{-1}}^{(0,1)}\left(t^{-1} u\right)} \\
& +\left|q^{\lambda_{1}} u\right\rangle_{\mathcal{V}_{q}} \otimes \sum_{i=1}^{l(\lambda)-1} \delta\left(q^{\lambda_{i+1}-1} t^{1-i} \frac{t^{-1} u}{z}\right) A_{\lambda \backslash \lambda_{1}, i}^{-}\left|\lambda \backslash \lambda_{1}-\square_{i}, t^{-1} u\right\rangle_{\mathcal{F}_{q, t^{-1}}^{(0,1)}\left(t^{-1} u\right)}
\end{aligned}
$$


As in the computation for $\psi^{-}(z)$ it is essential to ensure that when $\left[\lambda_{1}-1, \lambda \backslash \lambda_{1}\right]$ is not a Young diagram, the corresponding term cancels in eq. (A.8). This can happen when $\lambda_{1}=\lambda_{2}$ and precisely in this case the delta function in the third line of eq. (A.8) cancels with the function in the fourth line, which contains the factor

$$
\left(1-\frac{t}{q} q^{\lambda_{2}} \frac{t^{-1} u}{z}\right) \stackrel{\lambda_{2}=\lambda_{1}}{\rightarrow}\left(1-q^{\lambda_{1}-1} \frac{u}{z}\right) .
$$

All other "regular" cases, when $\left[\lambda_{1}-1, \lambda \backslash \lambda_{1}\right]$ is a Young diagram do not require extra cancellations and can be verified by comparing eqs. (A.6) and (A.8) term by term.

Thus, $\tilde{\Phi}_{q, t^{-1}}^{* q}$ is indeed the intertwiner acting from $\mathcal{F}_{q, t^{-1}}^{(0,1)}(u)$ to $\mathcal{V}_{q} \otimes \mathcal{F}_{q, t^{-1}}^{(0,1)}\left(t^{-1} u\right)$, as claimed in eq. (2.4).

Open Access. This article is distributed under the terms of the Creative Commons Attribution License (CC-BY 4.0), which permits any use, distribution and reproduction in any medium, provided the original author(s) and source are credited.

\section{References}

[1] H. Nakajima, Instantons on ALE spaces, quiver varieties, and Kac-Moody algebras, Duke Math. J. 76 (1994) 365 [INSPIRE].

[2] H. Nakajima, Heisenberg algebra and Hilbert schemes of points on projective surfaces, Annals Math. 145 (1997) 379.

[3] H. Nakajima, Quiver varieties and Kac-Moody algebras, Duke Math. J. 91 (1998) 515.

[4] L.F. Alday, D. Gaiotto and Y. Tachikawa, Liouville Correlation Functions from Four-dimensional Gauge Theories, Lett. Math. Phys. 91 (2010) 167 [arXiv:0906.3219] [INSPIRE].

[5] N. Wyllard, $A(N-1)$ conformal Toda field theory correlation functions from conformal $N=2$ $\mathrm{SU}(N)$ quiver gauge theories, JHEP 11 (2009) 002 [arXiv:0907.2189] [INSPIRE].

[6] A. Mironov and A. Morozov, On AGT relation in the case of U(3), Nucl. Phys. B $\mathbf{8 2 5}$ (2010) 1 [arXiv:0908.2569] [INSPIRE].

[7] Y. Zenkevich, Higgsed network calculus, JHEP 08 (2021) 149 [arXiv:1812.11961] [INSPIRE].

[8] J.-t. Ding and K. Iohara, Generalization and deformation of Drinfeld quantum affine algebras, Lett. Math. Phys. 41 (1997) 181 [INSPIRE].

[9] K. Miki, $A(q, \gamma)$ analog of the $W_{1+\infty}$ algebra, J. Math. Phys. 48 (2007) 123520.

[10] H. Awata and H. Kanno, Instanton counting, Macdonald functions and the moduli space of D-branes, JHEP 05 (2005) 039 [hep-th/0502061] [INSPIRE].

[11] A. Iqbal, C. Kozcaz and C. Vafa, The refined topological vertex, JHEP 10 (2009) 069 [hep-th/0701156] [INSPIRE].

[12] H. Awata, B. Feigin and J. Shiraishi, Quantum Algebraic Approach to Refined Topological Vertex, JHEP 03 (2012) 041 [arXiv:1112.6074] [INSPIRE].

[13] Y. Zenkevich, $\mathfrak{g l}_{N}$ Higgsed networks, arXiv:1912.13372 [InSPIRE]. 
[14] T. Kimura and V. Pestun, Quiver W-algebras, Lett. Math. Phys. 108 (2018) 1351 [arXiv: 1512.08533] [INSPIRE].

[15] T. Kimura and V. Pestun, Quiver elliptic W-algebras, Lett. Math. Phys. 108 (2018) 1383 [arXiv:1608.04651] [INSPIRE].

[16] T. Kimura and V. Pestun, Fractional quiver W-algebras, Lett. Math. Phys. 108 (2018) 2425 [arXiv: 1705.04410] [INSPIRE].

[17] D. Gaiotto and M. Rapčák, Vertex Algebras at the Corner, JHEP 01 (2019) 160 [arXiv:1703.00982] [INSPIRE].

[18] T. Procházka and M. Rapčák, Webs of W-algebras, JHEP 11 (2018) 109 [arXiv:1711.06888] [INSPIRE].

[19] T. Procházka and M. Rapčák, $\mathcal{W}$-algebra modules, free fields, and Gukov-Witten defects, JHEP 05 (2019) 159 [arXiv:1808.08837] [InSPIRE].

[20] M. Rapčák, Y. Soibelman, Y. Yang and G. Zhao, Cohomological Hall algebras, vertex algebras and instantons, Commun. Math. Phys. 376 (2019) 1803 [arXiv:1810.10402] [INSPIRE].

[21] M. Rapčák, On extensions of $\mathfrak{g l}(\widehat{m \mid n)}$ Kac-Moody algebras and Calabi-Yau singularities, JHEP 01 (2020) 042 [arXiv:1910.00031] [INSPIRE].

[22] M. Rapčák, Y. Soibelman, Y. Yang and G. Zhao, Cohomological Hall algebras and perverse coherent sheaves on toric Calabi-Yau 3-folds, arXiv:2007.13365 [INSPIRE].

[23] D. Gaiotto and M. Rapčák, Miura operators, degenerate fields and the M2-M5 intersection, arXiv:2012.04118 [INSPIRE]

[24] M. Bershtein, B. Feigin and G. Merzon, Plane partitions with a "pit": generating functions and representation theory, Sel. Math. New Ser. 24 (2018) 21 [arXiv:1512.08779].

[25] A. Mironov, A. Morozov and Y. Zenkevich, Ding-Iohara-Miki symmetry of network matrix models, Phys. Lett. B 762 (2016) 196 [arXiv:1603.05467] [INSPIRE].

[26] H. Awata et al., Explicit examples of DIM constraints for network matrix models, JHEP 07 (2016) 103 [arXiv: 1604.08366] [INSPIRE].

[27] J.-E. Bourgine, M. Fukuda, Y. Matsuo, H. Zhang and R.-D. Zhu, Coherent states in quantum $\mathcal{W}_{1+\infty}$ algebra and qq-character for 5d Super Yang-Mills, PTEP 2016 (2016) 123B05 [arXiv: 1606.08020$]$ [INSPIRE].

[28] J.-E. Bourgine, M. Fukuda, K. Harada, Y. Matsuo and R.-D. Zhu, $(p, q)$-webs of DIM representations, $5 d \mathcal{N}=1$ instanton partition functions and qq-characters, JHEP 11 (2017) 034 [arXiv: 1703.10759] [INSPIRE].

[29] J.-E. Bourgine, Webs of Quantum Algebra Representations in $5 d \mathcal{N}=1$ Super Yang-Mills, Springer Proc. Math. Stat. 263 (2017) 209 [INSPIRE].

[30] J.-E. Bourgine, M. Fukuda, Y. Matsuo and R.-D. Zhu, Reflection states in Ding-Iohara-Miki algebra and brane-web for D-type quiver, JHEP 12 (2017) 015 [arXiv:1709.01954] [INSPIRE].

[31] J.E. Bourgine and K. Zhang, A note on the algebraic engineering of $4 D \mathcal{N}=2$ super Yang-Mills theories, Phys. Lett. B 789 (2019) 610 [arXiv:1809.08861] [INSPIRE].

[32] J.-E. Bourgine, Fiber-base duality from the algebraic perspective, JHEP 03 (2019) 003 [arXiv: 1810.00301] [INSPIRE]. 
[33] B. Feigin, E. Feigin, M. Jimbo, T. Miwa and E. Mukhin, Quantum continuous $\mathfrak{g l}_{\infty}$ : Semi-infinite construction of representations, Kyoto J. Math. 51 (2011) 337 [arXiv: 1002.3100].

[34] H. Awata et al., Toric Calabi-Yau threefolds as quantum integrable systems. $\mathcal{R}$-matrix and $\mathcal{R} \mathcal{T} \mathcal{T}$ relations, JHEP 10 (2016) 047 [arXiv: 1608.05351] [INSPIRE].

[35] H. Awata et al., Anomaly in RTT relation for DIM algebra and network matrix models, Nucl. Phys. B 918 (2017) 358 [arXiv:1611.07304] [InSPIRE].

[36] H. Awata, H. Kanno, A. Mironov, A. Morozov, K. Suetake and Y. Zenkevich, The MacMahon R-matrix, JHEP 04 (2019) 097 [arXiv: 1810.07676] [INSPIRE].

[37] A. Negut, The R-matrix of the quantum toroidal algebra, arXiv:2005.14182 [INSPIRE].

[38] B. Feigin, M. Jimbo, T. Miwa and E. Mukhin, Finite Type Modules and Bethe Ansatz for Quantum Toroidal $\mathfrak{g l}_{1}$, Commun. Math. Phys. 356 (2017) 285 [arXiv: 1603.02765] [InSPIRE].

[39] B. Feigin, M. Jimbo, T. Miwa and E. Mukhin, Quantum toroidal $\mathfrak{g l}_{1}$ and Bethe ansatz, J. Phys. A 48 (2015) 244001 [arXiv: 1502.07194] [INSPIRE].

[40] S.M. Khoroshkin and V.N. Tolstoy, Universal R-matrix for quantized (super)algebras, Commun. Math. Phys. 141 (1991) 599.

[41] V.N. Tolstoy and S.M. Khoroshkin, The universal R-matrix for quantum untwisted affine Lie algebras, Funct. Anal. Appl. 26 (1992) 69.

[42] A. Hanany and E. Witten, Type IIB superstrings, BPS monopoles, and three-dimensional gauge dynamics, Nucl. Phys. B 492 (1997) 152 [hep-th/9611230] [INSPIRE].

[43] A. Nedelin, S. Pasquetti and Y. Zenkevich, $T[\mathrm{SU}(N)]$ duality webs: mirror symmetry, spectral duality and gauge/CFT correspondences, JHEP 02 (2019) 176 [arXiv:1712.08140] [INSPIRE].

[44] F. Aprile, S. Pasquetti and Y. Zenkevich, Flipping the head of $T[\mathrm{SU}(N)]$ : mirror symmetry, spectral duality and monopoles, JHEP 04 (2019) 138 [arXiv:1812.08142] [INSPIRE].

[45] M. Fukuda, Y. Ohkubo and J. Shiraishi, Non-Stationary Ruijsenaars Functions for $\kappa=t^{-1 / N}$ and Intertwining Operators of Ding-Iohara-Miki Algebra, SIGMA 16 (2020) 116 [arXiv: 2002.00243] [INSPIRE].

[46] B. Assel, Hanany-Witten effect and $\mathrm{SL}(2, \mathbb{Z})$ dualities in matrix models, JHEP 10 (2014) 117 [arXiv: 1406.5194] [INSPIRE].

[47] K. Costello, Supersymmetric gauge theory and the Yangian, arXiv:1303.2632 [INSPIRE].

[48] K. Costello, E. Witten and M. Yamazaki, Gauge Theory and Integrability, I, ICCM Not. 06 (2018) 46 [arXiv: 1709.09993] [INSPIRE].

[49] K. Costello, E. Witten and M. Yamazaki, Gauge Theory and Integrability, II, ICCM Not. 06 (2018) 120 [arXiv: 1802.01579] [INSPIRE].

[50] K. Costello and M. Yamazaki, Gauge Theory And Integrability, III, arXiv:1908.02289 [INSPIRE].

[51] V.V. Bazhanov and S.M. Sergeev, Zamolodchikov's tetrahedron equation and hidden structure of quantum groups, J. Phys. A 39 (2006) 3295 [hep-th/0509181] [inSPIRE].

[52] P. Gavrylenko, M. Semenyakin and Y. Zenkevich, Solution of tetrahedron equation and cluster algebras, JHEP 05 (2021) 103 [arXiv: 2010.15871] [INSPIRE]. 